\title{
RANDOM WALKS. I
}

\author{
BY \\ DONALD S. ORNSTEIN
}

By a random walk we will mean sums of independent, identically distributed random variables. Recently Spitzer discovered a class of limit theorems for random walks on the integers (or the points with integer coordinates in Euclidean space) and important contributions to this theory were made later by Kesten. The original work was motivated to a great extent by potential theory. However if we look at the results solely from the point of view of probability theory, they are of very great interest because they are the first theorems to be discovered that are natural, deep, and hold for any random walk on the integers. This is very much in keeping with the original spirit of probability theory which tries to make precise statements about the outcome of an experiment with a minimum of knowledge about its mechanism.

The purpose of this paper is to generalize some of these theorems to random walks on the line and to introduce some new methods in the case of the integers.

We will restrict ourselves to the line although everything works in higher dimensions and the proofs even simplify considerably. From here on when we speak of a random walk on the line we will assume that it is nonarithmetic, i.e., it does not live on an arithmetic progression containing 0 .

(A) Let $h_{x}(A, B)$ be the probability of hitting $A$ before $B$ starting at $x$. If $A$ and $B$ are finite intervals, then $\lim _{x \rightarrow \infty} h_{x}(A, B)$ and $\lim _{x \rightarrow-\infty} h_{x}(A, B)$ exists.

The above theorem implies that the distribution of the first hit in an interval tends to a limit as the starting point tends to $+\infty$ or $-\infty$ and this distribution can be shown to be nonsingular with respect to Lebesgue measure. In the transient case this is essentially the Blackwell-Feller-Orey renewal theorem. $\left[h_{x}(A, B)+\right.$ $h_{x}(B, A)=h_{x}(A \cup B, \phi)$. This and the nonsingularity of the distribution of the first hit gives us that the expected number of times we hit the interval $(x, x+1)$ starting at 0 tends to a limit as $x \rightarrow \infty$ and $x \rightarrow-\infty]$.

We get a variant of (A) by "reversing" the walk, namely: Let $H_{x}(A, B)$ be the expected number of times we hit $\mathrm{A}$ before hitting $B$, starting at $x$. If $A$ and $B$ are finite intervals, then $\lim _{y \rightarrow \pm \infty} H_{x}(A+y, B)$ both exist. This is also analogous to the renewal theorem which states (in our terminology): $\lim _{y \rightarrow \pm \infty} H_{x}(A+y, \phi)$ both exist.

Our method gives a new proof of (A) in the integer case and of the renewal theorem in this transient case. The essential ideas in the proof of $(A)$ are contained in $\$ 1$ where we prove a slightly weaker theorem. $\S 2$ contains a proof of (A) and also a proof of the variant mentioned above.

Received by the editors December 16, 1967. 
(B) For our next result we must assume that the distribution $u$ of our random walk has a nonsingular part (otherwise there are counterexamples).

If $A$ and $B$ are intervals of the same length, then starting at $x$ the difference between the expected number of times we visit $A$ and $B$ up to time $N$ tends to a limit as $N \rightarrow \infty$. Furthermore, if we call this limit $l(x)$, then $\lim _{x \rightarrow \pm \infty} l(x)$ exist. Also

$$
\lim _{x \rightarrow \infty} l(x)=-\lim _{x \rightarrow-\infty} l(x)= \pm\left(\int x\left(\psi_{A}-\psi_{B}\right)\right)\left(\int x^{2} u\right)^{-1} .
$$

A variant of the above theorem is the following: Let $u$ be a probability measure with a nonsingular part and let $T$ be convolution by $u$. Let $f(x)$ be a bounded Baire function with bounded support such that $\int f(x)=0$. Then

(1) $\sum_{i=1}^{n} T^{i} f$ converges pointwise (and in $L_{1}$ on finite intervals) to a bounded function $l(x)$.

(2) $\lim _{x \rightarrow \pm \infty} l(x)$ exist and

$$
\lim _{x \rightarrow \infty} l(x)=-\lim _{x \rightarrow-\infty} l(x)= \pm\left(\int x f\right)\left(\int x^{2} u\right)^{-1} .
$$

In the above form the theorem is analogous to the renewal theorem. It is also a strengthening (in this special case) of the ergodic theorem which says

$$
\frac{\sum_{i=1}^{n} T^{i} f^{+}}{\sum_{i=1}^{n} T^{i} f^{-}}
$$

converges pointwise ( $f^{+}$and $f^{-}$are the positive and negative parts of $f$ ).

As a corollary of the above theorem we get a picture of what $\sum_{i=1}^{n} u^{(i)}$ looks like for large $n$. If we denote by $v^{\prime \prime}$ the absolutely continuous part of the measure $v$, then there are constants $C_{n}$ such that $\left(\sum_{i=1}^{n} u^{(i)}\right)^{\prime \prime}-C_{n}$ converges (in $L_{1}$ on any finite interval) to a function $l(x)\left(C_{n}=\int_{0}^{1} \sum_{i=1}^{n} u^{(i)}\right)$. To complete the description of $\sum u^{(i)}$ note that the singular part of $\sum_{i=1}^{\infty} u^{(i)}$ is a finite measure. We can also describe the general shape of $l(x)$ in the following sense

$$
\begin{aligned}
\lim _{x \rightarrow \infty}\left[\int_{x}^{x+a} l(x)-\int_{x+b}^{x+b+a} l(x)\right] & =-\lim _{x \rightarrow-\infty}\left[\int_{x}^{x+a} l(x)-\int_{x+b}^{x+b+a} l(x)\right] \\
& = \pm a b\left(\int x^{2} u\right)^{-1 / 2}, \text { for any } a, b .
\end{aligned}
$$

It is also true that $\lim _{x \rightarrow \pm \infty} \int_{x}^{x+a} l(x)$ exist and that either both limits are $-\infty$ or one is $-\infty$ and the other finite.

These results are proved in $\S 3$. The main tools are the theorem in $\S 1$ and T2 of $\S 3$ which is a kind of ergodic theorem and holds for general Markov processes. The proofs in this section are independent of $\$ 2$.

(C) A random walk on the line is transient if and only if

$$
\int_{-a}^{a} \operatorname{Re}\left(\frac{1}{1-\hat{u}(\theta)}\right) \mathrm{d} \theta<\infty \quad \text { for some } a \neq 0 \text {. }
$$


( $u$ is a probability measure equal to the distribution of the random variables of the random walk and $\hat{u}(\theta)$ is its Fourier transform.) This simplifies the classical condition for transience due to Chung and Fuchs i.e.,

$$
\lim _{t>1} \int_{-a}^{a} \operatorname{Re}\left(\frac{1}{1-t \hat{u}(\theta)}\right) d \theta<\infty
$$

We prove (C) in $\S 4$. We do this by first proving (C) when $u$ has a nonsingular part and then using the idea of Stone and Port of reducing the general case to this special case. The proof will depend on T3.2 but will be independent of $\S 1, \S 2$ and the rest of $\S 3$.

(D) In $\S 5$ we will show that the results in $\$ 3$ can be used to show the existence and uniqueness of certain equations from potential theory. We assume here that $u$ has an absolutely continuous part. We then have: Let $r \geqq 0$ be a bounded Borel measurable function with bounded support, let

$$
l_{n}=C_{n}-\sum_{i=0}^{n} u^{(i)}
$$

$\left(C_{n}=\int_{0}^{1} \sum_{i=1}^{n} u^{(i)}, u^{(0)}=\delta_{0}, u^{(1)}=u\right.$ etc. $)$. Let $l=\lim _{n \rightarrow \infty} l_{n}\left(l_{n}^{\prime} \rightarrow l^{\prime}\right.$ by the results in $\S 3$ and the singular parts of $l_{n}$ obviously converge). Then there is a constant $K$ such that $g=l * r+K \geqq 0$ everywhere and $u * g=g+r$.

The idea of this proof is essentially the same as in the case of the integers. The proof of the next theorem is more interesting and gives something new and simpler in the case of the integers. Assume $u$ is recurrent and has an absolutely continuous component. Let $\dot{f}, g$ and $\psi$ be nonnegative Borel measurable functions. Let $\psi$ be bounded with bounded support. Then if $T f-f=T g-g=\psi$, it follows that $f-g$ $=a x+b$ a.e. ( $a$ and $b$ are constants). If $\int x^{2} u=\infty$ then $f-g=b$. (If $u$ is transient the theorem is still true if we replace $x$ by some one exponential depending on $u$. This however is already known.)

NotAtion. We will introduce the notation as we go along and try to repeat the definition when it may be helpful. We will however collect the main points of notation here for easy reference.

$u$ will always be a probability measure on the Borel sets of the line. $u$ will always be the distribution of the random variables governing the random walk.

If $v$ is a Borel measure on the line, $T v$ will be the Borel measure $u * v$.

If $f$ is a bounded Borel measurable function, $T f$ will be the Borel measurable function $u * f$.

$u^{(n)}$ will be $u$ convoluted by itself $n$ times.

All functions will be Borel measurable.

We will let $\int f=\int_{-\infty}^{\infty} f(x) d x$. If $v$ is a Borel measure we will let $\int f \cdot v$ be the integral of $f$ with respect to $v$.

$\delta_{x}$ will be the probability measure concentrated at $x$.

$\psi_{A}$ will be the function that is 1 on $A$ and 0 elsewhere.

$f^{1}=1-f$. 
$\bar{f}$ will be the operator: multiplication by the function $f$. (There are really two operators here, one sends Borel functions into Borel functions and the other sends Borel measures into Borel measures. Which one $\bar{f}$ stands for will be clear from the context.)

If $I=(a, b)$ then $I_{t}=(a+t, b-t),{ }^{t} I=(a-t, b+t)$.

$h_{x}(A, B)=$ the probability we hit $A$ before $B$ starting at $x$ (we will only use this for $A \cap B=\varnothing$; if $x \in A, h_{x}(A, B)=1$, if $\left.x \in B, h_{x}(A, B)=0\right)$.

$H_{x}(A, B)=\{$ the expected number of times we hit $A$ before $B$ starting at $x\}$ $=\int_{A} \sum_{i=0}^{\infty}\left(\bar{\psi}_{B}^{\prime} T\right)^{i} \delta_{x}$.

$h_{v}(A, B)$ and $H_{v}(A, B)$ will be defined in the same way as $h_{x}(A, B)$ and $H_{x}(A, B)$ except that we start with distribution equal to the measure $v$.

$I+b={ }_{b} I=$ the interval $I$ translated by $b \cdot{ }_{b} f(x)=f(x-b)$ and ${ }_{b} v$ is the measure $v$ translated by $b$.

$\tilde{f}(x)=f(-x)$. Similarly if $v$ is a measure $\tilde{v}$ is $v$ reflected around $0 . \tilde{T}$ is convolution by $u$.

$v^{\prime \prime}$ is the Radon-Nikodym derivative of the absolutely continuous part of the measure $v$.

$E \underline{T}=T \bar{\psi}_{E}^{\prime}$ (this will be used very seldom).

In $\S \S 0,1$, and $2, B$ will denote a subset of the line. In $\S \S 3,4$, and $5, B$ will denote a real number ( $B$ for bound). We point this out although it is clear from the context what is meant.

\section{Preliminaries.}

Let $f$ be a Borel measurable function (Baire function) and let $u$ be a probability measure defined on the Borel sets. Define $u * f$ as $u * f(x)=\int f(x-t) \cdot d u$. (We will sometime write $\int f d u=\int f \cdot u$.)

THEOREM 1. $u * f$ is a Borel measurable function.

Proof. 1. It is easy to see that it is enough to prove T1 when $f=\psi_{B}$ where $B$ is a Borel set and $\psi_{B}$ the function that is 1 on $B$ and 0 elsewhere.

2. If $\mathrm{T} 1$ is true for a countable family of disjoint sets $B_{i}$ then $\mathrm{T} 1$ is true for their union.

3. If $\mathrm{T} 1$ is true for a set $B$ then $\mathrm{T} 1$ is true for $\widetilde{B}$, the complement of $B$.

4. $\mathrm{T} 1$ is true when $B$ is an interval.

5. $\mathrm{T} 1$ is true for open and closed sets.

6. T1 is true for $O \cap C$ where $O$ is an open set and $C$ a closed set, $\left(\psi_{O \cap C}=\right.$ $\left.\psi_{0}-\psi_{O \cap \tilde{c}}\right)$.

7. T1 is true for any finite combination of open and closed sets using $\cup, \cap$ and $\sim$ (any such combination can be expressed as a finite union of disjoint sets each of which is the intersection of an open and closed set). In other words $\mathrm{T} 1$ is true for an algebra of sets including the open (and closed) sets.

8. Let $\mathscr{A}$ be a maximal algebra of sets including the open sets such that $\mathrm{T} 1$ is true. 
9. Consider the algebra $\overline{\mathscr{A}}$ generated by the collection $\mathscr{B}$ of all countable disjoint unions of sets in $\mathscr{A}$. Any set in $\overline{\mathscr{A}}$ can be written as a finite union of disjoint sets of the form: (a set in $\mathscr{B}) \cap$ (the complement of a set in $\mathscr{B})$. T1 is true for such a set since if $B_{1}$ and $B_{2}$ are in $\mathscr{B}, \psi_{B_{1} \cap \tilde{B}_{2}}=\psi_{B_{1}}-\psi_{B_{1} \cap B_{2}}$.

10. We conclude that $\mathscr{A}=\overline{\mathscr{A}}$ and hence that $\mathscr{A}$ is closed under countable unions and $\sim$. Hence $A$ includes the Borel sets and T1 is true for the Borel sets.

We would like next to define $u * v$ for two probability measures on the Borel sets. Let $F(x)$ be the distribution function of $v$. Then define $u * v$ to be the measure whose distribution function is $u * F$. [What we are actually doing is this: We define convolution of point measures in the obvious way. We approximate the continuous part of $u$ and $v$ by point masses (do not change the rest) by dividing the line into disjoint intervals $J_{i}$ each of length $<1 / n$ and replace the mass of the continuous part of $u$ (or $v$ ) in $J_{i}$ by a point mass in $J_{i}$. Call this $u_{n}\left(\right.$ or $\left.v_{n}\right)$. Then $\lim _{n \rightarrow \infty} u_{n} * v_{n}(-\infty, x]$ exists for each $x$ and we extend this set function to a measure (note it is right continuous).]

To each Borel measurable function $f$ there corresponds a Borel measure that assigns to $E, \int_{E} f$. Call this measure $\hat{f}$. It is then true that $(f \hat{*} v)=\hat{f} * v$ ( $v$ a Borel measure). This is obvious when $v$ is a point mass and we could approximate the continuous part of $v$ by point masses as we did above. The right side will converge to $\hat{f} * v$ by definition. The left side will give a sequence of functions which will converge in $L_{1}^{\prime}$ to $f * v$. To see this we write $f$ as a continuous function with compact support, $f_{1}$, plus a function with small $L_{1}$ norm, $f_{2}$, and we can then choose a sequence of continuous functions with compact support, $l_{i}$, such that $\sum_{i=1}^{\infty} l_{i}$ has small $L_{1}$ norm, $l_{i} \geqq 0$ and $\sum_{i=1}^{\infty} l_{i}>\left|f_{2}\right|$.

THEOREM 2. $\int(u * v) \cdot f=\int(\tilde{u} * f) \cdot v$. ( $\tilde{u}$ is the measure $u$ reflected around the origin. $u$ and $v$ are probability measures on the Borel sets and $f$, a Borel measurable function.)

Proof. The proof is almost the same as tha of T1. It is enough to prove T2 for $f=\psi_{E}$ where $E$ is any Borel set ( $\psi_{E}$ is 1 on $E$ and 0 elsewhere). If T2 is true for $\psi_{E_{i}}, E_{i}$, disjoint then T2 is true for $\psi_{\cup_{E_{i}}}$ and for $\psi_{\tilde{E}_{i}}$ and if $E_{1} \subset E_{2}$ and T2 is true for $\psi_{E_{2}}$ and for $\psi_{E_{1}}$ then T2 is true for $\psi_{E_{2} \cap \tilde{E}_{1}}$. We can repeat the same argument as in T1 as soon as we show that T2 is true for $\psi_{I}$ when $I$ is an interval. If we approximate the continuous part of $u$ by point masses as before then $\int u_{n} * v \cdot \psi_{I} \rightarrow \int u * v \cdot \psi_{I}$, $\int u_{n} * v \cdot \psi_{I}=\int \tilde{u}_{n} * \psi_{I} \cdot v$ and $\int \tilde{u}_{n} * \psi_{I} \cdot v \rightarrow \int \tilde{u} * \psi_{I} \cdot v$. The last equation comes from the fact that $\tilde{u}_{n} * \psi_{I}$ converges uniformly to $\tilde{u} * \psi_{I}$.

Let $X_{i}$ be a sequence of independent identically distributed random variables with distribution $u$ and let $T$ be the operator convolution by $u$. Let $\bar{g}$ be the operator multiplication by the function $g$ and let $\delta_{x}$ be the probability measure concentrated on $x$. Let $S_{n}=x+X_{1}, \ldots, X_{n}$. Then

$$
\operatorname{Pr}_{x}\left\{S_{n} \in A_{n}, n=1, \ldots, N\right\}=\int\left(\bar{\psi}_{A_{N}} T\right) \cdots\left(\bar{\psi}_{A_{1}} T\right) \delta_{x} .
$$


(This is easy to see and so we omit a proof. However, for our purposes, we could forget about random variables and use the right side of (1) to formalize the statement: the random walk, starting at $x$, is in the set $A_{n}$ at time $n$.)

THEOREM 3. If we denote the right side of $(1)$ by $f(x)$ then $f(x)$ is Borel measurable and $\int f \cdot v=\int\left(\bar{\psi}_{A_{N}} T\right) \cdots\left(\bar{\psi}_{A_{1}} T\right) v$ ( $v$ is a probability measure on the Borel sets).

Proof.

$$
\begin{aligned}
f(x) & =\int \psi_{A_{N}} \cdot\left(T \cdots \psi_{A_{1}} T\right) \delta_{x} \\
& =\int\left(\tilde{T} \psi_{A_{1}} \cdots \bar{\psi}_{A_{N-1}} \tilde{T}\right) \psi_{A_{N}} \cdot \delta_{x}=\left[\left(\tilde{T} \bar{\psi}_{A_{1}} \cdots \bar{\psi}_{A_{N-1}} \tilde{T}\right) \psi_{A_{N}}\right](x) .
\end{aligned}
$$

We get the middle equation by repeated use of $\mathrm{T} 1$ and $\mathrm{T} 2$. The other two are just definitions. (1) shows that $f(x)$ is Borel measurable (using T1 again). From (1) we get that

$$
\int f \cdot v=\int\left(\tilde{T} \psi_{A_{1}} \cdots \bar{\psi}_{A_{N-1}} \tilde{T}\right) \psi_{A_{N}} \cdot v
$$

We next repeat the argument in (1) to get

$$
\begin{aligned}
\int\left(\bar{\psi}_{A_{N}} T\right) \cdots\left(\bar{\psi}_{A_{1}} T\right) v & =\int \psi_{A_{N}} \cdot\left(T \cdots \bar{\psi}_{A_{1}} T\right) v \\
& =\int\left(\tilde{T} \bar{\psi}_{A_{1}} \cdots \bar{\psi}_{A_{N}-1} \tilde{T}\right) \psi_{A_{N}} \cdot v .
\end{aligned}
$$

We will give next, a few consequences of T3. Let us denote the function $1-g$ by $g^{\prime}$. Let us define

$$
h_{x}(A, B)=\int \sum_{i=0}^{\infty} \bar{\psi}_{A}\left(T \psi_{A \cup B}^{\prime}\right)^{i} \delta_{x}
$$

$h_{x}(A, B)$ is the probability that we hit $A$ before $B$ starting at $x$. (To avoid unnecessary confusion we will only consider the case where $A \cap B=\varnothing$.) T3 implies that $h_{x}(A, B)$ is a Borel measurable function if $A$ and $B$ are Borel sets.

Corollary 1. Let $D$ be a set containing $A \cup B, x \notin D$. Then $h_{x}(A, B)$ $=\int h_{y}(A, B) \cdot \rho$ where $\rho$ is the measure $\sum_{i=1}^{\infty} \bar{\psi}_{D}\left(T \bar{\psi}_{D}^{\prime}\right)^{i} \delta_{x}$ ( $\rho$ is the distribution of the first hit in $D$ starting at $x$ ).

This is completely obvious. Nevertheless we will give a formal proof not using random variables.

Proof. We will first show

$$
\left(T \bar{\psi}_{A \cup B}^{\prime}\right) v=T \bar{\psi}_{D}^{\prime} v+T \bar{\psi}_{A \cup B}^{\prime} \bar{\psi}_{D} v .
$$

To see (1) write $v=\bar{\psi}_{D} v+\bar{\psi}_{D}^{\prime} v$ and note that $\bar{\psi}_{A \cup B}^{\prime} \bar{\psi}_{D}^{\prime}=\bar{\psi}_{D}^{\prime}$. We use (1) to show by 
induction that

$$
\left(T \bar{\psi}_{A \cup B}^{\prime}\right)^{n} \delta_{x}=\left(T \bar{\psi}_{D}^{\prime}\right)^{n} \delta_{x}+\sum_{i=0}^{n-1}\left(T \bar{\psi}_{A \cup B}^{\prime}\right)^{n-i} \psi_{D}\left(T \bar{\psi}_{D}^{\prime}\right)^{i} \delta_{x},
$$

[(2) checks for $n=1$ by (1). Use (1) also to get

$$
\left(T \psi_{A \cup B}^{\prime}\right)\left(T \bar{\psi}_{D}^{\prime}\right)^{n} \delta_{x}=\left(T \bar{\psi}_{D}^{\prime}\right)^{n+1} \delta_{x}+\left(T \bar{\psi}_{A \cup B}^{\prime}\right) \psi_{D}\left(T \psi_{D}^{\prime}\right)^{n} \delta_{x}
$$

Now apply $T \psi_{A \cup B}^{\prime}$ to both sides of (2) to show that (2) is true for $n+1$.]

Apply $\psi_{A}$ to both sides of (2) and sum to get

(3) $\sum_{n=1}^{\infty} \bar{\psi}_{A}\left(T \bar{\psi}_{A \cup B}^{\prime}\right)^{n} \delta_{x}=\sum_{n=0}^{\infty} \bar{\psi}_{A}\left(T \bar{\psi}_{D}^{\prime}\right)^{n} \delta_{x}+\sum_{n=1}^{\infty} \sum_{i=0}^{n=1} \psi_{A}\left(T \bar{\psi}_{A \cup B}^{\prime}\right)^{n-i} \psi_{D}\left(T \bar{\psi}_{D}^{\prime}\right)^{i} \delta_{x}$.

(4) The right side of (3) is equal to

$$
\sum_{n=1}^{\infty} \sum_{i=0}^{n} \psi_{A}\left(T \psi_{A \cup B}^{\prime}\right)^{n-i} \psi_{D}\left(T \psi_{D}^{\prime}\right)^{i} \delta_{x}
$$

because $\psi_{A} \psi_{D}=\bar{\psi}_{A}$.

(3), (4) and the definition of $h_{x}(A, B)$ give

$$
h_{x}(A \cup B)=\sum_{n=0}^{\infty} \Psi_{A}\left(T \psi_{A \cup B}\right)^{n} \rho
$$

T3 and (5) give Corollary 1.

Corollary 2. Suppose $A \cup B+t \subset D, x+t \notin D$ for all $|t| \leqq 2$. Then

$$
\int_{x}^{x+1} h_{y}(A, B) d y=\int h_{y}(A, B) \cdot\left(\rho * \psi_{I}\right) d y
$$

where $I=(0,1)$ and $\rho$ is the same as in Corollary 1 .

by T3.)

$$
\left(\int_{x}^{x+1} h_{y}(A, B) d y=\int \sum_{i=0}^{\infty} \psi_{A}\left(T \psi_{A \cup B}^{\prime}\right)^{i} \psi_{(x, x+1)}\right)
$$

Proof. This is also obvious but we will give a formal proof which is a slight modification of that of Corollary 1 . Let $\mathscr{C}$ be the operator convolution by $\psi_{(0,1)}$. We then have

$$
\left(T \bar{\psi}_{A \cup B}^{\prime}\right) \mathscr{C} v=\mathscr{C}\left(T \bar{\psi}_{D}^{\prime}\right) v+T \bar{\psi}_{A \cup B}^{\prime} \mathscr{C} \bar{\psi}_{D} v
$$

To see (1) let $\mathscr{C} v=\mathscr{C} \psi_{D}^{\prime} v+\mathscr{C} \psi_{D} v, T \bar{\psi}_{A \cup B}^{\prime} \mathscr{C} \psi_{D}^{\prime} v=T \mathscr{C} \psi_{D}^{\prime} v$. Since $\bar{\psi}_{A \cup B}^{\prime} \mathscr{C} \bar{\psi}_{D}^{\prime}=\mathscr{C} \bar{\psi}_{D}^{\prime}$

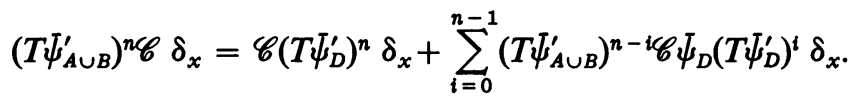

As before the case $n=1$ follows from (1), also by (1)

$$
\left(T \psi_{A \cup B}^{\prime}\right) \mathscr{C}\left(T \psi_{D}^{\prime}\right)^{n} \delta_{x}=\mathscr{C}\left(T \psi_{D}^{\prime}\right)^{n+1} \delta_{x}+T \psi_{A \cup B}^{\prime} \mathscr{C} \psi_{D}\left(T \psi_{D}^{\prime}\right)^{n} \delta_{x}
$$


From (2) we get

$$
\begin{aligned}
\sum_{n=1}^{\infty} \bar{\psi}_{A}\left(T \bar{\psi}_{A \cup B}^{\prime}\right)^{n \mathscr{C}} \delta_{x}= & \sum_{n=1}^{\infty} \bar{\psi}_{A} \mathscr{C}\left(T \bar{\psi}_{D}^{\prime}\right)^{n} \delta_{x} \\
& +\sum_{n=1}^{\infty} \sum_{i=0}^{n-1} \bar{\psi}_{A}\left(T \bar{\psi}_{A \cup B}^{\prime}\right)^{n-i} \mathscr{C} \bar{\psi}_{D}\left(T \bar{\psi}_{D}^{\prime}\right)^{i} \delta_{x} \\
= & \sum_{n=1}^{\infty} \sum_{i=0}^{n} \bar{\psi}_{A}\left(T \bar{\psi}_{A \cup B}^{\prime}\right)^{n-i} \mathscr{C} \bar{\psi}_{D}\left(T \bar{\psi}_{D}^{\prime}\right)^{i} \delta_{x} .
\end{aligned}
$$

The last equality comes from $\bar{\psi}_{A} \mathscr{C} \bar{\psi}_{D}=\bar{\psi}_{A} \mathscr{C},\left(\bar{\psi}_{A} \mathscr{C}=\bar{\psi}_{A} \mathscr{C} \bar{\psi}_{D}+\bar{\psi}_{A} \mathscr{C} \bar{\psi}_{D}^{\prime}\right.$ and $\left.\bar{\psi}_{A} \mathscr{C} \bar{\psi}_{D}^{\prime}=0\right)$ (3) and T3 imply Corollary 2.

Corollary 3. Pick sets $E, F, D$, a measure $v$ whose support is disjoint from $D$ and a positive linear operator $\mathscr{C}$ that commutes with $T$. Assume that $E$ and $F$ are in $D$ and that the support of $\mathscr{C} \bar{\psi}_{D}^{\prime}$ is disjoint from $E \cup F$. Then

$$
\sum_{n=1}^{\infty} \bar{\psi}_{F}\left(T \bar{\psi}_{E}^{\prime}\right)^{n} \mathscr{C} v=\sum_{n=0}^{\infty} \bar{\psi}_{F}\left(T \bar{\psi}_{E}^{\prime}\right)^{n} \quad\left(\sum_{i=0}^{\infty} \mathscr{C}_{D}\left(T \bar{\psi}_{D}^{\prime}\right)^{i} v\right) .
$$

Proof. The proof is exactly the same as that of (3) Corollary 2 and will be omitted.

If $u$ is a measure on the Borel sets on the line we will denote by $u^{(n)}$ the convolution of $u$ with itself $n$ times. We will say that $u$ is arithmetic if the support of $u$ is an arithmetic progression containing 0 . It is then easy to see that if $u$ is nonarithmetic and if $\sum_{i=1}^{\infty} u^{(i)}$ is infinite for some interval it is infinite for all intervals (see Feller [2, p. 200]). (If $\sum_{i=1}^{\infty} u^{(i)}$ is infinite on some interval we say that the random walk, given by random variables with distribution $u$, is recurrent. If the walk is not recurrent we say it is transient.)

From now on we will assume $u$ to be nonarithmetic. We can strengthen the assertion in the previous paragraph if we start with an absolutely continuous distribution instead of at a point as follows:

THEOREM 4. If $u$ is nonarithmetic, recurrent and $f$ a bounded nonnegative, integrable Borel measurable function, $\int f>0$, then $\int_{E} \sum_{i=1}^{\infty} u^{(i)} * f=\infty$ for any Borel set $E$ of nonzero measure.

\section{Proof.}

$$
\int_{E}\left(\sum_{i=1}^{\infty} u^{(i)}\right) * f=\int\left(\sum_{i=1}^{\infty} u^{(i)}\right) * f \cdot \psi_{E}=\int \sum_{i=1}^{\infty} u^{(i)} \cdot \psi_{E} * \tilde{f}
$$

$\tilde{f} * \psi_{E}$ is continuous and therefore $\tilde{f} * \psi_{E}>\varepsilon \psi_{I}$ for some $\varepsilon>0$ and some interval $I$.

If $u$ is recurrent (and nonarithmetic) then if $E$ is a Borel set of nonzero measure we will hit $E$ infinitely often with probability 1 for almost every starting point $x$ and from every starting point $x$ if $u$ has an absolutely continuous part. The theorem below is equivalent to this. (However the equivalence is not necessary for our purposes since we will only use the version in the theorem.) 
THEOREM 5. Let $u$ be recurrent (and nonarithmetic), f a nonnegative Borel measurable function and $E$ a Borel set of nonzero measure. Let ${ }_{E} \underline{T}$ be the operator that agrees with convolution by $u$ on functions with support outside of $E$ and sends functions with support in $E$ into 0 . We then have

$$
\int_{E} \sum_{n=0}^{\infty}{ }_{E} \underline{T}^{n} f=\int f\left(\text { where }_{E} \underline{T}^{0} f=f\right) .
$$

Proof. Assume the theorem is false. We then have

$$
\int_{E} \sum_{n=0}^{\infty}{ }_{E} \underline{T}^{n} f<C \int f \text { for some } C<1 .
$$

Without loss of generality we could assume that the support of $f$ is disjoint from $E$.

(1) There is a set $B$ of nonzero measure, such that $B \cap E=\varnothing$ and for any $g \geqq 0$ with support in $B$ we have

(a) $\int_{E} \sum_{n=0}^{\infty} E \underline{T}^{n} g<C^{\prime} \int g$ where $C^{\prime}=C+\frac{1}{2}(1-C)$. To prove (1): let $\alpha_{1}=\sup \int g$ where sup is taken over all $g \geqq 0, g \leqq f$ and equation (a) is false. Pick $g_{1}$ such that $\int g_{1}>\frac{1}{2} \alpha_{1}, f \geqq g_{1} \geqq 0$ and (a) is false for $g_{1}$. Next let $f_{1}=f-g_{1}, \alpha_{2}=\sup \int g$ sup taken over all $f_{1} \geqq g \geqq 0$ and (a) is false for $g$. Pick $g_{2}$ such that $\int g_{2}>\frac{1}{2} \alpha_{2}, f_{1} \geqq g_{2} \geqq 0$ and (a) is false for $g_{2}$. Continue this procedure defining $g_{1} g_{2} \cdots g_{n}$ etc. Let $f_{\infty}=f$ $-\sum_{i=1}^{\infty} g_{i} \cdot \int f_{\infty}>0$ since (a) is true for $f$ but false for $\sum_{i=1}^{\infty} g_{i}$. Let $B$ be a set such that for some $\varepsilon>0, f_{\infty}(x)>\varepsilon$ for $x \in B$. We now have that (a) is true for any $g \geqq 0$, $g \leqq \varepsilon$, support of $g$ in $B$, but $g \leqq \varepsilon$ is irrelevant and hence we have (1).

(2) There is a set $E^{\prime} \subset E$, of nonzero measure, an integer $N$ and $\varepsilon>0$ such that (a) $\int_{B} T^{N} g>\varepsilon \int g$ for any $g \geqq 0$ with support in $E^{\prime}$.

To see (2): We can find some $\varepsilon$ and $N$ such that $\int_{B} T^{N} f>2 \varepsilon \int f$ where $f=\psi_{E}$ by T4. We next proceed exactly as in (1) picking a sequence $g_{i}$ such that (a) is false for each $g_{i}$ and each $g_{i}$ is almost as large as possible subject to the condition that $\sum_{n=1}^{\infty} g_{n}<f$.

(3) There is a constant $K$ such that if $g$ has support in $E^{\prime}$ then $\int_{E^{\prime}} \sum_{i=0}^{\infty}{ }_{B} \underline{T}^{i} g$ $<K \int g$. We will get (3) from (2) as follows: If $k$ is a function let $\bar{k}$ be the operator multiplication by $k$ and $k^{\prime}=1-k$ then ${ }_{B} \underline{T}=T \bar{\psi}_{B}^{\prime}$

$$
\left(T \bar{\psi}_{B}^{\prime} \sum_{i=0}^{\infty}\left(T \bar{\psi}_{B}^{\prime}\right)^{i} g\right)=\bar{\psi}_{B}^{\prime} \sum_{i=1}^{\infty}\left(T \bar{\psi}_{B}^{\prime}\right)^{i} g+l
$$

where $l=\bar{\psi}_{B} \sum_{i=1}^{\infty}\left(T \bar{\psi}_{B}^{\prime}\right)^{i} g$. All that we need to know about $l$ however is that $\int l \leqq \int g$. Let us write $\bar{\psi}_{B}^{\prime} \sum_{i=0}^{\infty}\left(T \bar{\psi}_{B}^{\prime}\right)^{i} g=h$. From (a) we get

$$
T^{N} h \leqq h+\sum_{i=0}^{N} T^{i} l
$$

Next note that $h \geqq \bar{\psi}_{E^{\prime}} \sum_{i=0}^{\infty}\left(T \bar{\psi}_{B}^{\prime}\right)^{i} g=h_{1}$ since $E \cap B=\varnothing$. Apply (2) to $h_{1}$ and use the above inequality to get

$$
\varepsilon \int h_{1}<\int_{B} T^{N} h_{1} \leqq \int_{B} T^{N} h
$$


combine (c) with (b) to get

$$
\varepsilon \int h_{1} \leqq \int_{B} h+N \int l
$$

(d) is what we want since

$$
\int l \leqq \int g, \quad \int h_{1}=\int_{E^{\prime}} \sum_{i=0}^{\infty}\left({ }_{B} \underline{T}^{i}\right) g \text { and } \int_{B} h=0
$$

(4) We will now get a contradiction by showing that if $g$ has support in $E^{\prime}$ then $\int_{E^{\prime}} \sum_{i=0}^{\infty} T^{i} g<\infty$. To see (4) we first note:

$$
\left(\sum_{i=0}^{\infty} T^{i} g\right) \psi_{E^{\prime}}=\left(\sum_{j=1}^{\infty} \sum_{i=0}^{\infty}{ }_{B} \underline{T}^{i} h_{j}\right) \psi_{E^{\prime}}
$$

where $h_{1}=g, f_{j}=\left(\sum_{i=0 B}^{\infty} \underline{T}^{i} h_{j}\right) \psi_{B}, h_{j+1}=\left(\sum_{i=0}^{\infty} E \underline{T}^{i} f_{j}\right) \psi_{E^{\prime}}$. (a) is easy to see and we omit a proof. It is also easy to see that $\int_{E^{\prime}} \sum_{n=0}^{\infty} E_{E^{\prime}} \underline{T}^{n} g \leqq \int_{E} \sum_{n=0}^{\infty} E \underline{T}^{n} g$ and therefore (1) implies that $\int h_{j+1} \leqq C^{\prime} \int f_{j}$ and from this we get

$$
\int h_{j+1} \leqq C^{\prime} \int h_{j}
$$

(3) implies

(c)

$$
\int_{E^{\prime}} \sum_{i=0}^{\infty}{ }_{B} \underline{T}^{i} h_{j}<K \int h_{j}
$$

(a), (b) and (c) now show that $\int_{E^{\prime}} \sum_{i=0}^{\infty} T^{i} g<\infty$.

THEOREM 6. Let $u$ be recurrent and $f$ a Borel measurable function such that $f(x) \geqq 0$ and $u * f=f$ a.e. Then there is a constant $C$ such that $f(x)=C$ a.e.

Proof. Assume T6 is false. We may also assume without loss of generality that both $\int(f-1)^{+} \neq 0$ and $\int(f-1)^{-} \neq 0$.

(1) $u *(f-1)=f-1$ a.e. Let $g=(l-1)^{+}$and $h=(f-1)^{-}$so that $f-1=g-h$. Note that $h(x) \leqq 1$. Let us rewrite (1) as follows.

(2) $u *(g-h)=g-h+e$ everywhere where $e$ is a Borel measurable function $\int|e|=0$ (e=0 a.e.).

(3) $u^{(n)} *(g-h)=g-h+\sum_{i=0}^{n-1} u^{(i)} * e$.

(4) $u^{(n)} * h \geqq h$ a.e. This is so because $g$ is 0 on the support of $h$ and on the support of $h$ we have by (4)

$$
u^{(n)} * h-u^{(n)} * g=h \sum_{i=0}^{n-1} u^{(i)} * e .
$$

By choosing $u$ properly we can get that there is a set $E$ of nonzero measure such that both $u^{(n)} * g(x)>0$ and $h(x)>0$ for $x \in E$. This gives us

(5) $u^{(n)} * h-h>0$ on a set of nonzero measure. 
(6) Let $v=u^{(n)}$ and let $s=u^{(n)} * h-h$, then $v * h=h+s^{+}+s^{-}$and $\int s^{-}=0$ by (4).

(7) $v^{(i)} * h=h+\sum_{j=0}^{i-1} v^{(j)} s^{+}+\sum_{j=0}^{i-1} v^{(j)} s^{-}$.

Since $h(x) \leqq 1, v^{(i)} * h(x) \leqq 1$ but for any interval $I$

$$
\lim _{i \rightarrow \infty} \int_{I} \sum_{j=0}^{i-1} v^{(j)} * s^{+}=\infty
$$

by (5) and $\mathrm{T} 4$ and $\int \sum_{j=0}^{i-1} v^{(j)} * s^{-}=0$ giving a contradiction.

As before let $\psi_{E}$ be the function that is 1 on $E$ and 0 elsewhere let $E+a$ be the set $E$ translated by $a$ and let $\delta_{x}$ be the probability measure concentrated at the point $x$. Let $u^{(n)}$ be $u$ convoluted by itself $n$ times.

THEOREM 7. (a) Let $u$ be nonarithmetic. Then given an interval I and numbers a and $\varepsilon>0$ we can find integers $n$ and $k$ such that $\int\left|u^{(n)} * \psi_{1}-u^{(n+k)} * \psi_{I+a}\right|<\varepsilon$.

(b) If we rule out the possibility that the support of $u$ lies in an arithmetic progression $\left({ }^{1}\right)$, then

$$
\lim _{n \rightarrow \infty} \int\left|u^{(n)} *\left(\psi_{I}-\psi_{I+a}\right)\right|=0
$$

(c) If $u$ has an absolutely continuous part, then

$$
\lim _{n \rightarrow \infty} \int\left|u^{(n)} *\left(\delta_{0}-\delta_{a}\right)\right|=0 .
$$

(d) If the support of $u$ lies in an arithmetic progression containing 0 and if $a$ is in the group generated by the support of $u$ then

$$
\lim _{n \rightarrow \infty} \int\left|u^{(n)} *\left(\delta_{0}-\delta_{a}\right)\right|=0
$$

(e) In both (b) and (c) the convergence is uniform for all $a$ in a bounded interval (in (b) keep 1 fixed).

It is easy to see that (b) will remain true if we replace $\psi_{I}-\psi_{I+a}$ by any Borel measurable function $g$ of integral 0 since we could approximate $g$ in the $L_{1}$ norm by sum of function of the first type. However we will not use this.

Proof. We will first prove (b). Pick $\varepsilon>0$. Then pick random variables $X_{1}$ and $Y_{1}$ both with distribution $u$ such that $Z_{1}=X_{1}-Y_{1} \neq 0$ is bounded and the group generated by the range of $Z_{1}$ contains a number of absolute value $<\varepsilon$. (To do this we first pick $X_{1}$. Then we let $Y_{1}=X_{1}$ on the set where $\left|X_{1}\right|>N$ for some very large $N$. We then pick $Y$ to have the same distribution as $X_{1}$ on this part of the measure space $\Omega$, and to be independent of $X_{1}$ on the rest of $\Omega$. If $N$ is chosen large enough we get our desired $Z_{1}$.) Next pick a sequence $Z_{n}=X_{n}-Y_{n}$ such that any two $Z_{i}$ have the same distributions and any two of the $X_{i}$ or $Y_{i}$ have the same distribution, and

(1) Recall that nonarithmetic means that the support of $u$ does not lie in an arithmetic progression containing 0 . 
such that $Z_{n}$ (and $X_{n}$ and $Y_{n}$ ) are independent of everything that came before. Now the $\{Z\}$ give a recurrent random walk and so we will hit an interval of length $2 \varepsilon$ around $-a$ with probability 1 . (This means that the random walk governed by $\sum X_{\imath}$ and the walk governed by $a+\sum Y_{i}$ come within $2 \varepsilon$ of each other with probability 1.) Let $F_{n}$ be the set where $\left|a-\sum_{i=1}^{n} Z_{i}\right| \leqq 2 \varepsilon$ and $\left|a-\sum_{i=1}^{j} Z_{i}\right|>2 \varepsilon$ for $j<n$. It is easy to see that we can replace the sequence $X_{i}$ and $Y_{i}$ by sequences $X_{i}^{\prime}$ and $Y_{i}^{\prime}$ so that $X_{n}^{\prime}=Y_{n}^{\prime}$ on $\bigcup_{i=1}^{n-1} F_{i}$ and $X_{n}^{\prime}=X_{n}$ and $Y_{n}^{\prime}=Y_{n}$ on the rest of $\Omega$ and $X_{n}^{\prime}$ and $Y_{n}^{\prime}$ are independent. (This means that the walks given by $\sum X_{n}^{\prime}$ and $a+\sum Y_{n}^{\prime}$ get and stay within $2 \varepsilon$ of each other with probability 1.) This last fact enables us (for $n$ large enough) to write the distribution of $\sum_{i=1}^{n} X_{i}^{\prime}, u^{(n)}(u$ convoluted by itself $n$ times) as the sum of positive measures $u_{i}(i=1, \ldots, K)$ and to write the distribution of $a+\sum_{i=1}^{n} Y_{i}^{\prime}, u^{(n)} * \delta_{a}$ as the sum of positive measures $\bar{u}_{i}(i=1, \ldots, K)$ in such a way that $\int u_{i}=\int \bar{u}_{i}(i=1, \ldots, K)$ and $u_{i}+\bar{u}_{i}$ has support in an interval of length less than $4 \varepsilon(i=2, \ldots, K)$ and $\int u_{1}<\varepsilon$. This shows that

$$
\int\left|\psi_{I} *\left(u^{n}-u^{(n)} * \delta_{a}\right)\right|=\int\left|u^{(n)} *\left(\psi_{I}-\psi_{I+a}\right)\right|
$$

can be made as small as we want by making $\varepsilon$ small enough. This gives (b). To get (c) we note that if $k$ is large enough $u^{(k)}$ can be written as the sum of 2 nonnegative measures $u_{1}$ and $u_{2}$ where $\int u_{2}$ can be made as small as we like and $u_{1}(E)=\int_{E} g$ for all Borel sets $E$ where $g$ is continuous $(g \geqq 0)$. This shows as before that

$$
\int\left|u^{(k)} *\left(u^{(n)}-u^{(n)} * \delta_{a}\right)\right|
$$

can be made as small as we want by making $k$ and $n$ large enough.

To get (e) it is enough to show that for cach of the integrals in (b) and (c) and for $\varepsilon>0$ we can find an $N$ and $\delta$ such that the integral is $<\varepsilon$ for all $n>N$ and all $|a|<\delta$ ( $n=N$ will do since both integrals are monotone nonincreasing in $n$ ). This is obvious for the integral in (b) and for the integral in (c) we simply write $u^{(k)}=u_{1}+u_{2}$ as before.

To get (d) we repeat the proof of (b) except that the group generated by the range of $Z_{1}$ should be the same as the group generated by the support of $u$ (it can no longer contain an element of absolute value $<\varepsilon$ ).

To get (a) we need only consider the case where the support of $u$ lies in an arithmetic progression not containing 0 . Then $u=u_{1} * \delta_{b}$ where the support of $u_{1}$ lies in an arithmetic progression containing 0 . Without loss of generality we could assume that the group generated by the support of $u_{1}$ is the integers and that $b$ is irrational. Pick integers $k$ and $n$ such that $|N-(a+b k)|<\varepsilon$. Let $c=N-(a+b k)$. Let $v=\delta_{c} * u^{(k)} * \delta_{a}$. It is clear that $v$ has support on the integers. By (d)

$$
\lim _{n \rightarrow \infty} \int\left|u^{(n)} *\left(v-\delta_{0}\right)\right|=\lim _{n \rightarrow \infty} \int\left|u_{1}^{(n)} *\left(v-\delta_{0}\right)\right|=0 .
$$


Rewriting the last equation we have

$$
\lim _{n \rightarrow \infty} \int\left|u^{(n+k)} * \delta_{a+c}-u^{(n)} * \delta_{0}\right|=0,
$$

and this easily gives (a).

\section{Section 1}

This section contains a proof of Theorem 1. The proof is written for the recurrent case and the changes necessary for the transient case are put in such a way that the reader who wants to ignore them can. The reason for this is that in the transient case we can get the theorem as a consequence of the Blackwell-FellerOrey renewal theorem. The remarks on the transient case were added for the sake of a unified approach, and to provide another proof of the renewal theorem.

Definition. Let $h_{x}(A, B)$ be the probability of hitting $A$ before $B$ starting at $x$ $(A \cap B=\varnothing)$.

THEOREM 1. For a nonarithmetic random walk on the line and two fixed bounded, Borel sets $A$ and $B$,

both exist.

$$
\lim _{t \rightarrow \infty} \int_{t}^{t+1} h_{x}(A, B) d x \text { and } \lim _{t \rightarrow-\infty} \int_{t}^{t+1} h_{x}(A, B) d x
$$

L1. For each $\varepsilon>0$ and $M>0$ there exists $N>0$ such that if $|y|>N$ and $|a|<M$ then

$$
\left|\int_{y}^{y+1} h_{x}(A, B)-\int_{y+a}^{y+a+1} h_{x}(A, B)\right|<\varepsilon
$$

Proof.

$$
\int_{y}^{y+1} h_{x}(A, B)=\int_{A} \sum_{i=0}^{\infty}\left[T\left(\overline{1-\psi}_{A \cup B}\right)\right]^{i} \psi_{(y, y+1}
$$

by T0.3 (Theorem 3 in $\S 0$ ). ( $\psi_{D}$ is the function that is 1 on $D$ and 0 elsewhere, $\psi_{(y, y+1)}$ is 1 on the interval $(y, y+1)$ and 0 elsewhere. $\bar{g}$ is the operator multiplication by the function $g$. $T$ is the operator convolution by $u$ where $u$ is a probability measure equal to the distribution of the random variables governing the walk.) It is easy to see that for fixed $n$

$$
\lim _{y \rightarrow \infty} \int\left|\left[T\left({\overline{1-\psi_{A \cup B}}}\right)\right]^{n} \psi_{(y, y+1)}-T^{n} \psi_{(y, y+1)}\right|=0
$$

We can now apply $\mathrm{T} 0.7$ to get $\mathrm{L} 1$.

\{If we make use of recurrence the proof is trivial and we outline it here. Note

$$
\int_{y}^{y+1} h_{x}(A, B) d x=\int_{0}^{-1} h_{y}(A+t, D+t) d t .
$$


Also if we start at $y$ we will hit an interval of length $\frac{1}{4} \varepsilon$ around $y+a$ with probability greater than $1-\frac{1}{4} \varepsilon$ before some time $N$. If $|y|$ is large enough we will hit $\bigcup_{|t|<1} A+t$ $\cup B+t$ with probability $<\frac{1}{4} \varepsilon$ before time $N$. These facts give L1. $\}$

Notation. Let $s=\lim \sup _{|t| \rightarrow \infty} \int_{t}^{t+1} h_{x}(A, B) d x$. Let $O_{\varepsilon}$ be the set of $t$ such that

$$
\left|\int_{t}^{t+1} h_{x}(A, B) d x-s\right|<\varepsilon \text {. }
$$

L2. Given $\varepsilon>0$ (and bounded Borel sets $A$ and $B$ ) there exists an $N$ such that if $|t|>N$ then either $t$ or $-t$ is in $O_{\varepsilon}$.

Proof. We will start by assuming that there are arbitrarily large $t$ such that $t$ and $-t$ are not in $O_{\varepsilon}$, and get a contradiction.

Let ${ }_{t} D$ stand for the set $D$ translated by $t$.

Pick an integer $M>10 / \varepsilon$. The first step in the proof will be to construct a set $E$. $E$ will be the union of translates of $A \cup B$ by numbers $t_{i}, i=1, \ldots, M$, and $E$ will have the property: (1) for each $i, i=1, \ldots, M$,

$$
\int_{y}^{y+1} h_{x}\left({ }_{t_{i}} A,{ }_{t_{i}} B\right) d x<s-\frac{9}{10} \varepsilon
$$

if for some $j, j \neq i$, the distance of $y$ from ${ }_{t_{j}} A \cup_{t} B$ is less than 1 .

We construct $E$ as follows: Let $t_{1}=0$ and let $E_{1}=A \cup B$. Assume now that we can construct a set $E_{n}$ consisting of $n$ translates of $A \cup B$ (by numbers $t_{i}, i=1, \ldots, n$ ) and satisfying (1). We will then get a set $E_{n+1}$ consisting of $n+1$ translates of $A \cup B$ satisfying (1). By assumption there are arbitrarily large $t$ such that

$$
\int_{t}^{t+1} h_{x}(A, B) d x<s-\varepsilon \text { and } \int_{0}^{1} h_{x}\left({ }_{t} A,{ }_{t} B\right) d x<s-\varepsilon .
$$

L1 tells us that if $t$ is large enough then $\int_{y}^{y+1} h_{x}\left({ }_{t} A,{ }_{t} B\right) d x<s-9 \varepsilon / 10$ if $y$ has distance less than 1 from $E_{n}$. Also

$$
\int_{y}^{y+1} h_{x}\left({ }_{t_{i}} A,{ }_{t_{i}} B\right) d x<s-\frac{9}{10} \varepsilon
$$

if $y$ has distance less than 1 from ${ }_{t} A \cup{ }_{t} B$, and $i=1, \ldots, n$. Call this $t, t_{n+1}$ and let $E_{n+1}=E_{n} \cup_{t_{n+1}} A \cup_{t_{n+1}} B$. Clearly $E_{n}$ satisfies (1). Let $E=E_{M}$.

By L1 we can pick a number $w$ such that

$$
\int_{w}^{w+1} h_{x}\left(l_{i} A, t_{i} B\right) d x>s-\frac{1}{10} \varepsilon \text { for } i=1, \ldots, M .
$$

Let $\rho$ be the distribution of the first place we hit $E$ starting at $w$. Let $\rho_{i}$ be the part of $\rho$ in ${ }_{t_{i}} A \cup{ }_{t_{i}} B, i=1, \ldots, M$. For some $i$ (call it $k$ ) we have:

$$
\int \rho_{k}<\frac{\varepsilon}{10} .
$$


By Corollary 2 of T0.3 we have

$$
\begin{aligned}
\int_{w}^{w+1} h_{x}\left(t_{k} A, t_{k} B\right) d x & =\int h_{x}\left(\left(_{t_{k}} A,{ }_{t_{k}} B\right) \cdot\left(\rho * \psi_{I}\right) d x\right. \\
& =\int h_{x}\left(t_{t_{k}} A,{ }_{t_{k}} B\right) * \tilde{\psi}_{I} \cdot \rho d x \\
& =\sum_{i=1}^{M} \int h_{x}\left(t_{t_{k}} A,{ }_{t_{k}} B\right) * \tilde{\psi}_{I} \cdot \rho_{i} d x,
\end{aligned}
$$

where $I=(0,1)$ and $\tilde{\psi}_{I}=\psi_{(-1,0)}$. (The second equality comes from T0.2.)

Because of (1) the function $h_{x}\left(t_{k} A,{ }_{t_{k}} B\right) * \tilde{\psi}_{I}$ is less than $s-9 \varepsilon / 10$ at all points of ${ }_{t_{i}} A \cup{ }_{t_{i}} B, i \neq k$. Therefore

$$
\int h_{x}\left({ }_{t_{k}} A,{ }_{t_{k}} B\right) * \tilde{\psi}_{I}, \rho_{i} d x<\left(s-\frac{9}{10} \varepsilon\right) \int \rho_{i} \text { for } i \neq k
$$

Combining (3), (4) and (5) we get

$$
\int_{w}^{w+1} h_{x}\left(t_{k} A,{ }_{t_{k}} B\right) d x<s-\frac{8}{10}
$$

but this contradicts (2).

Proof of Theorem. Let

$$
\bar{s}=\limsup _{|y| \rightarrow \infty} \int_{y}^{y+1} h_{x}(B, A)=1-\liminf _{|y| \rightarrow \infty} \int_{y}^{y+1} h_{x}(A, B) .
$$

Let $\bar{O}_{\varepsilon}$ be the set of $y$ such that $\left|\int_{y}^{y+1} h_{x}(B, A)-\bar{s}\right|<\varepsilon$. We would then have L2 with $O_{\varepsilon}$ replaced by $\bar{O}_{\varepsilon}$. These two versions of L2 imply that for large enough $y$ either $y$ is in $O_{\varepsilon}$ and $-y$ in $\bar{O}_{\varepsilon}$ or $-y$ is in $\bar{O}_{\varepsilon}$ and $y$ is in $O_{\varepsilon}$. If $s=1-\bar{s}$, we are finished, otherwise choose $\varepsilon<\frac{1}{4}[s-(1-\bar{s})]$. Therefore $y_{1}$ in $O_{\varepsilon}$ and $y_{2}$ in $\bar{O}_{\varepsilon}$ implies

$$
\left|\int_{y_{1}}^{y_{1}+1} h_{x}(A, B)-\int_{y_{2}}^{y_{2}+1} h_{x}(A, B)\right|>\frac{1}{2} \varepsilon
$$

Combining this with L1 we see that for some $N$ we have $y>N \Rightarrow y$ is in $O_{\varepsilon}$ and $y<-N \Rightarrow y$ is in $\bar{O}_{\varepsilon}$ or $y>N \Rightarrow y$ is in $\bar{O}_{\varepsilon}$ and $y<-N \Rightarrow y$ is in $O_{\varepsilon}$.

\{In the transient case we get as far as L2 but the last paragraph does not work because L2 does not work if we replace $s$ by $s^{\prime}$ where

$$
s^{\prime}=\liminf _{|x| \rightarrow \infty} \int_{x}^{x+1} h_{y}(A, B) d y .
$$

We finish off the theorem as follows. Let $P_{x}(a, b)$ be the probability of hitting $(a, b)$ starting at $x$. Let $S_{\varepsilon}$ be the set of $x$ such that $P_{x}\left(-\frac{1}{2}, \frac{1}{2}\right)<\varepsilon$.

We will be finished if we can show that (1) for each $\varepsilon>0$ there is an $N$ such that if $|x|>N$ then $x$ or $-x$ is in $S_{\varepsilon}$. 
Before proving (1) we will prove

$$
\liminf _{|x| \rightarrow \infty}\left[\psi_{(1 / 2,1 / 2)} *\left(\sum_{i=1}^{\infty} u^{(i)}\right)\right](x)=0
$$

( $u$ is the probability measure equal to the distribution of the random walk and $u^{(t)}$ is the $i$ fold convolution of $u$ with itself.) To see (2) note that

$$
\lim _{k \rightarrow \infty}\left[u^{(k)} *\left(\psi_{(-1 / 2,1 / 2)} * \sum_{i=1}^{\infty} u^{(i)}\right)\right](0)=\lim _{k \rightarrow \infty} \int_{-1 / 2}^{1 / 2} \sum_{i=k}^{\infty} u^{(i)}=0 .
$$

The left side of the above equation equals

$$
\int\left(\psi_{(-1 / 2,1 / 2)} * \sum_{i=1}^{\infty} u^{(i)}\right) \cdot u^{(k)}
$$

and since for each fixed $N, \int_{-\infty}^{-N} u^{(k)}+\int_{N}^{\infty} u^{(k)}$ can be made as close to 1 as we want by making $k$ large enough, we get (2).

(2) implies (3) $\lim \inf _{|x| \rightarrow \infty} P_{x}(-1 / 2,1 / 2)=0$. (3) implies (1) because if (1) were false there would be an $\varepsilon>0$ and arbitrarily large $x$ such that $P_{0}\left(x-\frac{1}{2}, x+\frac{1}{2}\right)>\varepsilon$ and $P_{x}\left(-\frac{1}{2}, \frac{1}{2}\right)>\varepsilon$. For each fixed $y$ we can choose $x$ large enough so that the last inequality will imply (by L1) that $P_{x}\left(y-\frac{1}{2}, y+\frac{1}{2}\right)>\frac{1}{2} \varepsilon$. Hence $P_{0}\left(y-\frac{1}{2}, y+\frac{1}{2}\right)>\frac{1}{2} \varepsilon^{2}$ for all $y$ contradicting (3).

\section{Section 2}

In this section we will improve on and get some variants of T1.1. Our first goal will be to prove Theorem (A) in the introduction. (We do not assume recurrence here.)

Definition. Let $H_{x}(A, B)=\int_{A} \sum_{i=0}^{\infty}\left(\bar{\psi}_{B}^{\prime} T\right)^{i} \delta_{x}$ (to avoid unnecessary confusion we will only use this definition for $A \cap B=\varnothing)$. $H_{x}(A, B)$ is the expected number of times we hit $A$ before hitting $B$ starting at $x$. (See pp. 2-4 for the definition $\psi_{B}^{\prime}, T$ and $\left.\delta_{x}\right)$.

L1. $H_{x}(A, B)<\alpha<\infty$ where $\alpha$ is a constant independent of $x$ (it may depend on $A$ and $B)$ if $A$ and $B$ are intervals.

Proof. This is obvious in the transient case so we assume recurrence

$$
\int_{B} T \sum_{i=0}^{\infty}\left(\bar{\psi}_{B}^{\prime} T\right)^{i} \delta_{x} \leqq 1
$$

This term is simply the probability of hitting $B$ starting at $x$. (1) is formally obvious since

$$
\begin{gathered}
\int\left(\bar{\psi}_{B}^{\prime} T\right)^{n} \delta_{x}=\int_{B} T\left(\bar{\psi}_{B}^{\prime} T\right)^{n} \delta_{x}+\int\left(\bar{\psi}_{B}^{\prime} T\right)^{n+1} \delta_{x} . \\
\int_{B} T^{k} \sum_{i=0}^{\infty}\left(\bar{\psi}_{B}^{\prime} T\right)^{i} \delta_{x} \leqq K .
\end{gathered}
$$

(2) follows easily from (1). 
(3) Write $A$ as the union of disjoint interval $A_{i}$ each of length $<1 / 10$ the length of $B$ and with center $x_{i}$ and let $B^{\prime}$ be an interval with the same center as $B$ and of length $<1 / 10$ the length of $B$. Then using the recurrence we can get a $k_{i}$ and $\varepsilon_{i}>0$ for each $x_{i}$ such that $\int_{B^{\prime}} T^{k_{i}} \delta_{x_{i}}>\varepsilon_{i}$ and hence

(4) $\int_{B} T^{k_{i}} \delta_{x}>\varepsilon_{i}$ for any $x \in A_{i}$.

Now if L1 were false there would be an $A_{i}$ such that for arbitrarily large $N$ we could find an $x$ for which

(5) $\int_{A_{1}} \sum_{j=0}^{\infty}\left(\bar{\psi}_{B}^{\prime} T\right)^{j} \delta_{x}>N$.

Now it is clear using T0.3 that 4 and 5 contradict (2).

NotATION. If $I$ is an interval let $I_{t}$ and ${ }^{t} I$ be the intervals with the same center as $I$ and length $|I|-2 t$ and $|I|+2 t$ respectively $(|I|=$ length of $I)$.

L2. Fix an interval I and a point $y \notin I$. If $\left|x_{i}\right| \rightarrow \infty$ and $t_{i} \rightarrow 0, t_{i}>0$ then

$$
\lim _{i \rightarrow \infty} H_{x_{i}}\left(J_{i}, I\right)=0
$$

where $J_{i}=\left(y, y+t_{i}\right)$.

Proof. If $\mathrm{L} 2$ were false there would be an $\varepsilon>0$ such that $H_{x_{i}}\left(J_{i}, I\right)>\varepsilon>0$ for infinitely many $i$ and hence (1) for each $j, H_{x_{i}}\left(J_{j}, I\right)>\varepsilon$ for infinitely many $i$. Let $v_{i, j}$ be the measure whose Radon-Nikodym derivative is $1 / t_{j}$ on $\left(x_{i}-\frac{1}{2} t_{j}, x_{i}+\frac{1}{2} t_{j}\right)$ and 0 elsewhere. (1) implies that $H_{y}\left(t_{j} J_{j}, I_{t}\right)>\varepsilon$ for $x_{i}-\frac{1}{2} t_{j} \leqq y \leqq x_{i}+\frac{1}{2} t_{j}$ and we therefore get using T0.3 that for each $j$ there are infinitely many $i$ such that

$$
H_{v_{i},{ }^{\prime}}\left({ }^{t_{j}} J_{j}, I_{t,}\right)>\varepsilon \quad\left(H_{v}(A, B)=\int_{A} \sum_{i=0}^{\infty}\left(\bar{\psi}_{B}^{\prime} T\right)^{i} v\right) .
$$

Pick an interval $J$ with center $y, J \cap I=\varnothing$ and $s=|J|<|I| / 10$. By L1 there is an $\alpha$ such that

$$
H_{x}\left(J, I_{4 s}\right)<\alpha \text { for all } x .
$$

Pick an integer $N$ such that $N \varepsilon>4 \alpha$. Pick $n$ points $\beta_{i}$ in $J, i=1, \ldots, N$ and pick $l$ such that

$$
t_{l}<\frac{1}{10} \min _{i \neq j}\left|\beta_{i}-\beta_{j}\right| .
$$

Let $\gamma_{i}=\beta_{i}-y$, and let $\gamma_{i} v$ be $v$ translated by $\gamma_{i}$. We then apply T0.7 to (2) to get (4) for some $k$ (large enough)

and hence

$$
H_{y_{i} v_{k, l} l}\left(t_{l} J_{l}, I_{t_{l}}\right)>\frac{1}{2} \varepsilon \text { for } \gamma_{i}, i=1, \ldots, N,
$$

$$
H_{v_{k, l}}\left(t_{i} J_{l}+\gamma_{i}, I_{l+\gamma_{i}}\right)>\frac{1}{2} \varepsilon \text { for } \gamma_{i}, i=1, \ldots, N
$$

$(J+\gamma=J$ translated by $\gamma)$. Note that $t_{i} J_{l}+\gamma_{i} \cap t_{l} J_{l}+\gamma_{j}=\varnothing$ for $i \neq j, i, j=1, \ldots, N$ and $t_{l} J_{l}+\gamma_{i} \subset J, i=1, \ldots, N$. Note also that $I_{4 s} \subset \bigcap_{i=1}^{N} I_{l+\gamma_{i}}$ (5) therefore implies 
that $H_{v_{k, l}}\left(J, I_{4 s}\right)>\frac{1}{2} \varepsilon N$ but this (and T0.3) contradicts (3). We can now get Theorem (A) in the introduction namely

THEOREM 1. If $A$ and $B$ are finite intervals (with some, none, or all of their endpoints) then $\lim _{x \rightarrow \infty} h_{x}(A, B)$ and $\lim _{x \rightarrow-\infty} h_{x}(A, B)$ exist.

Proof. Let $B^{\prime}$ be some closed interval in the interior of $B$. Let $\zeta_{t}$ be the union of the four (or 3) intervals of length $t$ whose center is an endpoint of $A$ or $B$. L2 tells us that given $\varepsilon$ we can find a $t>0$ such that for all $x$ large enough we have $H_{x}\left(\zeta_{t}, B^{\prime}\right)$ $<\varepsilon$ and hence (1) $h_{x}\left(\zeta_{t}, B^{\prime}\right)<\varepsilon$ (for all $x$ large enough). (We can choose $t$ so that $\zeta_{t} \cap B^{\prime}=\varnothing$.) (1) implies (2) there is an $N$ such that if $|x|>N$ and $|x-y|<\frac{1}{2} t$ then $\left|h_{x}(A, B)-h_{y}(A, B)\right|<\varepsilon$. To see (2): $h_{y}(A, B)=h_{x}(A+(x-y), B+(x-y))$. We then use Corollary 1 to T0.3 to get $h_{x}(A+(x-y), B+(x-y))$ and $h_{x}(A, B)$ equal $\int h_{y}(A+(x-y), B+(x-y)) \rho$ and $\int h_{y}(A, B) \rho$ respectively where $\rho$ is the distribution of the first hit in $A \cup B \cup A+(x-y) \cup B+(x-y)$. The only part of $\rho$ which will contribute differently to the two integrals is the part of $\rho$ in $\zeta_{t}$ which is $<\varepsilon$ by (1).

We next combine (2) and T0.7 (Theorem 7, §0) to reduce T1 to T1.1 (Theorem 1, $\S 1)$. Break the interval $(x, x+1)$ into disjoint intervals $J_{i}$ of length $t . h_{y}(A, B)$ varies by $<\varepsilon$ on each $J_{i}$ if $|x|>N$, by (2). If $|x|$ is large enough T0.7 tells us that

$$
\left|\int_{J_{i}} h_{y}(A, B)-\int_{J_{j}} h_{y}(A, B)\right|<t \varepsilon .
$$

Hence $\left|h_{x}(A, B)-\int_{x}^{x+1} h_{y}(A, B)\right|<3 \varepsilon$.

THEOREM 2. For any nonarithmetic random walk on the line and bounded intervals $A$ and $B, \lim _{y \rightarrow \infty} H_{x}(B+y, A)$ and $\lim _{y \rightarrow-\infty} H_{x}(B+y, A)$ exist and are finite.

$\mathrm{T} 2$ will be a consequence of the following lemma:

L3. Let $\psi_{E}$ be the function that is 1 on $E$ and 0 elsewhere. Let $\psi_{E}^{\prime}=1-\psi_{E}$, let $\bar{f}$ be the operator multiplication by $f$ and let $A$ and $B$ be bounded intervals. Then for fixed $y$

$$
\sum_{i=1}^{\infty}\left(T \bar{\psi}_{A}^{\prime}\right)^{i} \psi_{B+y}=g_{y}(x)
$$

is a function defined for each $x . \lim _{y \rightarrow \infty} g_{y}(x)$ and $\lim _{y \rightarrow-\infty} g_{y}(x)$ exist for each $x$. $\left(g_{y}(x)\right.$ will be bounded on each finite interval.) The convergence is uniform for all $x$ in a finite interval.

Proof. Let $I$ be an interval containing $A$ and $B+A$. Let the measure on $I$, $\alpha_{y}=\left(\sum_{i=0}^{\infty}\left(T \psi_{I}\right)^{i} \delta_{y}\right) \cdot \psi_{I} .\left(\alpha_{y}\right.$ is the distribution of the position of the first hit in $I$ starting at $y$.)

It is easy to see that (Corollary 3 of T0.3)

$$
\sum_{i=0}^{\infty}\left(T \bar{\psi}_{A}^{\prime}\right)^{i} \psi_{B+y}=\sum_{i=0}^{\infty}\left(T \psi_{A}^{\prime}\right)^{i}\left(\alpha_{y} * \psi_{B}\right) \quad \text { on } J
$$

where $J$ is any interval such that $J$ and $J+B$ are in $I$. 
(2) Given $\varepsilon>0$ there is an $N$ such that if $y_{1}>N$ and $y_{2}>N$ then

$$
\left|\left(\alpha_{y_{1}} * \psi_{B}-\alpha_{y_{2}} * \psi_{B}\right)(x)\right|<\varepsilon
$$

on $I+B$ and 0 elsewhere. We get (2) by the following argument: By L2 there is a $\delta>0$ and $N_{1}$ such that if $L$ is any interval in $I$ of length $<\delta$ then $h_{y}(L, I-L)<\frac{1}{2} \varepsilon$ for $y>N_{1}$. Now write $I$ as the union of $L_{i}, i=1, \ldots, M, L_{i}$ being disjoint intervals of length $<\delta$. Next use T1 to pick $N>N_{1}$ such that for each $i$ and $y>N$ and $z>N$ we have $\left|h_{y}\left(L_{i}, I-L_{i}\right)-h_{z}\left(L_{i}, I-L_{i}\right)\right|<\varepsilon / 2 M$. (2) is now obvious.

(1) and (2) will give us L3 if we can show that

$$
\sum_{i=1}^{\infty}\left[\left(T \bar{\psi}_{A}^{\prime}\right)^{i} \psi_{K}\right](x)<b<\infty
$$

for all $x$ in a finite interval ( $b$ may depend on this interval) where $K$ is an interval containing $I$ and $I+B$. To see (3) break $K$ into the union of intervals $K_{i}, i=1, \ldots, M$ of length $t$ and center $x_{i}$. (Choose any $t$ such that $4 t<$ length of $A$.) We now have that for each $K_{i}$

$$
\left[\sum_{i=0}^{\infty}\left(T \bar{\psi}_{A}^{\prime}\right)^{i} \psi_{K_{i}}\right](x)<\left[\sum_{i=0}^{\infty}\left(T \bar{\psi}_{A_{t}}^{\prime}\right)^{i} \delta_{x_{i}} * \psi_{\left(K_{i}-x_{i}\right)}\right](x)
$$

for all $x$. The right side of the last equation is $<b$ for some $b$ and all $x$ because $\sum_{i=0}^{\infty}\left(T \bar{\psi}_{A}^{\prime}\right)^{i} \delta_{x_{i}}$ gives finite measure to any interval. (To get (4): let $\mathscr{C}$ be the operator convolution by $\psi_{K_{i}-x_{i}}$ and $v$ a Borel measure. Then we have $v=\psi_{A_{t}}^{\prime} v+\psi_{A_{t}} v$ and $\bar{\psi}_{A}^{\prime} \mathscr{C} v=\bar{\psi}_{A}^{\prime} \mathscr{C} \bar{\psi}_{A_{t}}^{\prime} v \leqq \mathscr{C} \bar{\psi}_{A_{t}}^{\prime} v$ and hence $\left(T \bar{\psi}_{A}^{\prime}\right) \mathscr{C} v \leqq \mathscr{C}\left(T \bar{\psi}_{A_{t}}^{\prime}\right) v$ and $\left(T \bar{\psi}_{A}^{\prime}\right)^{n} \mathscr{C} \delta_{x_{i}} \leqq \mathscr{C}\left(T \bar{\psi}_{A_{t}}^{\prime}\right)^{n} \delta_{x_{i}}$. The last inequality is (4).)

Proof of T2. $H_{x}(B+y, A)=\int\left(\sum_{i=0}^{\infty}\left(\psi_{A}^{\prime} T\right)^{i} \delta_{x}\right) \cdot \psi_{B+y}$. By T0.2 the right side of the last equation is equal to

$$
\left[\sum_{i=0}^{\infty}\left(\tilde{T} \psi_{A}^{\prime}\right)^{i} \psi_{B+y}\right](x)
$$

and by $\mathrm{L} 3$ this converges as $y \rightarrow \infty$ or $y \rightarrow-\infty$.

THEOREM 3. For all nonarithmetic walks on the line and bounded disjoint intervals $A$ and $B, \lim _{x \rightarrow \infty} H_{x}(A, B)$ and $\lim _{x \rightarrow-\infty} H_{x}(A, B)$ exist and are finite.

Proof. Pick an interval $B^{\prime}$ interior to $B$. Then given $\varepsilon$ use L2 to get a $t$ such that

(1) $\lim _{|x| \rightarrow \infty} H_{x}\left({ }^{t} A-A_{t}, B^{\prime}\right)<\varepsilon$ and

(2) $\lim _{|x| \rightarrow \infty} H_{x}\left({ }^{t} B-B_{t}, B^{\prime}\right)<\varepsilon^{\prime} \quad\left[\varepsilon^{\prime}\right.$ to be chosen later] (pick $t$ so that $B^{\prime} \subset B_{t}$ ). Our immediate goal will be to use (1), (2), and L1 to get that, for any $\varepsilon>0$,

(3) $\left|H_{x}(A, B)-H_{y}(A, B)\right|<2 \varepsilon$ for all $x$ large enough and $|x-y|<\frac{1}{2} t$. To do this write

(4) $\bar{\psi}_{B}^{\prime}=\bar{\psi}_{B \cup B+t}^{\prime}+\bar{\psi}_{B+t-B} .(B+t$ is $B$ translated by $t$ and $A-B$ is the part of $A$ not in $B$.) Then

$$
\left(\bar{\psi}_{B}^{\prime} T\right)^{n} \delta_{x}=\left(\bar{\psi}_{B \cup B+t}^{\prime} T\right)^{n} \delta_{x}+\sum_{i=0}^{n-1}\left(\bar{\psi}_{B}^{\prime} T\right)^{n-1-i}\left(\bar{\psi}_{B+t-B} T\right)\left(\bar{\psi}_{B+t \cup B}^{\prime} T\right)^{i} \delta_{x}
$$


we get (5) from (4) by induction

$$
\sum_{n=1}^{\infty}\left(\bar{\psi}_{B}^{\prime} T\right)^{n} \delta_{x}=\sum_{n=1}^{\infty}\left(\bar{\psi}_{B \cup B+t}^{\prime} T\right)^{n} \delta_{x}+\sum_{n=0}^{\infty}\left(\bar{\psi}_{B}^{\prime} T\right)^{n} \rho
$$

and

$$
\rho=\sum_{i=0}^{\infty}\left(\bar{\psi}_{B+t-B} T\right)\left(\bar{\psi}_{B+t \cup B}^{\prime} T\right)^{i} \delta_{x}
$$

(6) is still true if we interchange $B$ and $B+t$ call this equation $\left(6^{\prime}\right)$ and the corresponding $\rho, \rho^{\prime}$.

(2) implies that $\int \rho<\varepsilon^{\prime}$ and $\int \rho^{\prime}<\varepsilon^{\prime}$, for $x$ large enough. By L1 we get that if $\varepsilon^{\prime}$ is chosen small enough compared to $\varepsilon$ we have

$$
\int_{2 t_{A}} \sum_{i=0}^{\infty}\left(\bar{\psi}_{B}^{\prime} T\right)^{i} \rho<\frac{1}{2} \varepsilon \quad\left(\text { and } \int_{2 t_{A}} \sum_{i=0}^{\infty}\left(\bar{\psi}_{B+t}^{\prime} T\right)^{i} \rho^{\prime}<\frac{1}{2} \varepsilon\right) .
$$

6 and $6^{\prime}$ therefore give us

$$
\int_{2 t_{A}}\left|\sum_{n=1}^{\infty}\left(\bar{\psi}_{B}^{\prime} T\right)^{n} \delta_{x}-\sum_{n=1}^{\infty}\left(\bar{\psi}_{B+t}^{\prime} T\right)^{n} \delta_{x}\right|<\varepsilon
$$

( $x$ large enough).

(7) implies that (8) $\left|H_{x}(A, B)-H_{x-t}(A-t, B)\right|<\varepsilon$ for $x$ large enough. (1) and (8) give (3). (3) says that

$$
\lim _{x \rightarrow \infty} H_{x}(A, B)=\lim _{x \rightarrow \infty} \int_{x}^{x+1} H_{y}(A, B) d y
$$

and the limit on the right exists by L3.

THEOREM 4. Define $L(y)$ for $y$ in some interval $(a, b)$ to be $\lim _{x \rightarrow \infty} h_{x}((a, y),(y, b))$. Then there is an absolutely continuous measure $v$ such that $L(y)=v(a, y)$.

Proof. $L(y)$ is continuous by L2. Therefore $v$ is defined. Suppose there were a set $E$ of measure 0 such that $v(E)=\alpha>0$. By L2 we can pick $t$ such that

$$
\lim _{x \rightarrow \infty} h_{x}((a-t, a+t) \cup(b-t, b+t),(a+t, b-t))<\frac{1}{4} \alpha
$$

(we may assume $E \subset(a+t, b-t)$ ). Let $\rho_{s, x}=$ the distribution of the first hit in $(a+s, b+s)$ starting at $x$. For $x$ large enough and $|s|<t$ we have (1)

$$
\int\left|\rho_{s, x}-\rho_{0, x}\right|<\frac{1}{2} \alpha \text {. }
$$

(To see this let $\rho$ be the distribution of the first hit in $(a-s, b+s)$. Then

$$
\int\left|\rho-\rho_{0, x}\right|<\frac{1}{4} \alpha \text { and } \int\left|\rho-\rho_{s, x}\right|<\frac{1}{4} \alpha
$$

since we get $\rho_{s, x}$ from $\rho$ by replacing $\rho \psi_{(a-s, a+s)}$ by the distribution of the first hit in $(a+s, b+s)$ starting with distribution $\rho \psi_{(a-s, a+s)}$.) 
This implies that $\int\left|{ }_{s} v-v\right|<\frac{1}{2} \alpha$ for all $|s|<t\left({ }_{s} v=v\right.$ translated by $\left.s\right)$. This in turn implies that $\int v * \psi_{E} \neq 0$ which is a contradiction.

\section{Section 3}

In this section we will assume that our probability measure, $u$, has a nonsingular part. Let $T$ be convolution by $u$. Our main result is

THEOREM 1. Let $f(x)$ be bounded, with bounded support and $\int f=0$. Then (1) $\sum_{i=1}^{n} T^{i} f$ tends pointwise (and in $L_{1}$ on finite intervals) to a bounded function $\bar{f}(x)$ and (2) $\lim _{x \rightarrow \infty} \bar{f}(x)$ and $\lim _{x \rightarrow-\infty} \bar{f}(x)$ exist.

Notation. Recall that all functions that we consider are Baire functions and all measures Borel measures. We will let $\int f$ stand for $\int_{-\infty}^{\infty} f(x) d x$ and if $v$ is a measure we will let $\int_{E} v=v(E)$ ( $E$ a Borel set), and $\int v=v(-\infty, \infty), \int v f=\int f d v$, etc.

We will only give the proof of $\mathrm{T} 1$ in the recurrent case since in the transient case (1) is obvious and (2) is an immediate consequence of the renewal theorem (a proof which has been given in $\$ 1$ ).

The main tools in the proof of T1 will be T2.1 (Theorem $1, \S 2$ ) and T2 of this section. Note however that when $u$ has a nonsingular part T2.1 follows immediately from $\mathrm{T} 1.1$ because of $\mathrm{T} 0.7$.

For easy reference we will list below 3 simple lemmas. (L3 is an easy case of T0.2.)

L1. Let $f(x) \geqq 0, \int f(x)>0$. Then the convolution of $f$ with itself is bounded away from 0 on some interval.

Proof. This follows from the fact that the convolution of a bounded measurable function with itself is continuous.

L2. For any interval $I$ we can find an $n$ such that the Radon-Nikodym derivative of the nonsingular part of $u^{(n)}$ is bounded away from 0 on $I$. ( $u$ is assumed to be recurrent and to have a nonsingular part.)

L3. Let $\tilde{T}$ be convolution by $u(-x)$ and let $\delta_{x}$ be the probability measure concentrated at $x$. Then $(T f)(x)=\int f \circ\left(\tilde{T} \delta_{x}\right)$.

Before proving $\mathrm{T} 1$ we will prove $\mathrm{T} 2$.

TheORem 2. Let I be a finite interval. Then there is a constant $B$ such that for all $f$ with support in $I,|f(x)| \leqq 1$ and $\int f(x)=0$ we have $\left|\left(\sum_{i=1}^{n} T^{i} f\right)(x)\right|<B$ for all $x$ and $n$.

Remark. The conclusion of T2 is equivalent to $\left|\int f \circ\left(\sum_{i=1}^{n} T^{i} \delta_{x}\right)\right|<B$ for all $x$ and $n$ by L3.

Proof. Let $f=r-h$ where $r$ and $h$ are nonnegative and have disjoint support.

(A) We will start by defining two sequences of functions $r_{i}$ and $l_{i}$ :

Let $r_{1}=r$ and $l_{1}=0$, 
Let $r_{2}=T r_{1}-\inf \left(T r_{1}, h\right), l_{2}=\inf \left(T r_{1}, h\right)$,

Let $r_{3}=T r_{2}-\inf \left(T r_{2}, h-l_{2}\right), l_{3}=\inf \left(T r_{2}, h-l_{2}\right)$,

Let

$$
\begin{aligned}
& r_{i}=T r_{i-1}-\inf \left(T r_{i-1}, h-\sum_{j=1}^{i-1} l_{j}\right), \\
& l_{i}=\left(\inf T r_{i-1}, h-\sum_{j=1}^{i-1} l_{j}\right) .
\end{aligned}
$$

An equivalent way of defining the $r_{i}$ and $l_{i}$ is as follows: Define the (nonlinear) operator $K$ so that $K f=T f^{+}-f^{-}$. Then $r_{i}=\left(K^{i-1} r\right)^{+}$and $h-\sum_{j=1}^{i} l_{j}=\left(K^{i-1} r\right)^{-}$.

(B) The next step is to show that $\sum_{i=1}^{\infty} r_{i}$ has finite integral over any interval.

(1) $\int r_{n}=\int h-\int_{j=1}^{n} l_{j}$.

(2) For each $n$ there is a set $S_{n} \subset I$ of nonzero measure such that $\sum_{i=1}^{n} r_{i}(x)=0$, for $x \in S_{n}$ (or $r_{i}=0$ a.e. for $i>n$ in which case (B) is obvious).

This is so because $\sum_{i=1}^{n} l_{i}(x)<h(x)$ on a set of nonzero measure (or $\int \sum_{i=1}^{n} l_{i}=\int h$ and $r_{i}=0$ a.e. for $\left.i>n\right)$.

(3) $T\left(\sum_{i=1}^{n} r_{i}\right)=\sum_{i=2}^{n+1} r_{i}+\sum_{i=1}^{n+1} l_{i}$ and therefore

$$
T\left(\sum_{i=1}^{n} r_{i}\right)(x) \leqq 1, \quad x \in S_{n}
$$

(4) From (3) we get for fixed $K$ and every $n$ there is an $S_{n}^{\prime} \subset I$ such that

$$
T^{K}\left(\sum_{i=1}^{n} r_{i}\right)(x)<K \text { for } x \in S_{n}^{\prime} .
$$

(Choose $S_{n}^{\prime}$ so that $\sum_{i=1}^{n+k} r_{i}(x)=0$ for $x \in S_{n}^{\prime}$.)

We can now show that $\int_{J} \sum_{i=1}^{\infty} r_{1}<\infty$ for any finite interval $J$. Let $D$ be the maximum distance between a point in $I$ and a point in $J$ and let $H=(-D, D)$. Then by Lemma 2 there is an $\alpha>0$ and an integer $K$ such that the derivative of the absolutely continuous part of $T^{K} \delta_{0}>\alpha$ for $x \in H$.

(5) If $\int_{J} \sum_{i=1}^{n} r_{i}>2 K / \alpha$ then $T^{K}\left(\sum_{i=1}^{m} r_{i}\right)(x)>2 K$ for any $x \in I$ ( $\alpha$ and $K$ depend only on $I$ and $J$ ) by T0.3 but this would contradict (4).

(C) The next step is to show that $\sum_{i=1}^{\infty} r_{i}(x)<B$, a.e. We first show

(1) $\sum_{i=1}^{\infty} l_{i}=h$ a.e. $\left(\sum_{i=1}^{\infty} l_{i}^{\prime}=r\right.$ a.e. $)$.

This follows from the recurrence of the walk and T0.5 since if (1) were false then $\mathrm{a}^{\circ}$ there would be a set $E$ of nonzero measure and $\varepsilon>0$ such that $h(x)-\sum_{i=1}^{\infty} l_{i}(x)>\varepsilon$ for $x \in E$. This would imply that $\mathrm{b}^{\circ} r_{k}(x)=0, x \in E$. From $\mathrm{b}^{\circ}$ we get $\mathrm{c}^{\circ} r_{k} \leqq_{E} \underline{T}^{k} r$ $=\left(T \bar{\psi}_{E}^{\prime}\right)^{k} r\left(\bar{\psi}_{E}^{\prime}\right.$ is the operator: multiplication by the function that is 0 on $E$ and 1 elsewhere). We get $c^{\circ}$ by induction. Assume $c^{\circ}$ is true for $k$ then $r_{k} \leqq \bar{\psi}_{E}^{\prime}\left(\underline{T} \bar{\psi}_{E}^{\prime}\right)^{k} r$ by $b^{\circ}$ and $r_{k+1} \leqq T r_{k} \leqq\left(T \psi_{E}^{\prime}\right)^{k+1} r$. From $b^{\circ}$ and $c^{\circ}$ we get $d^{\circ} \int r_{k} \leqq \int_{E^{\prime}}{ }_{E} T^{k} r\left(E^{\prime}=\right.$ complement of $E$ ). By $\mathrm{a}^{\circ}$ and (B)(1) there is an $\alpha>0$ such that $\int r_{k}>\alpha$ for all $k$ hence by $\mathrm{d}^{\circ}$ $\int_{E^{\prime} E} \underline{T}^{k} r>\alpha>0$ for all $k$ but this contradicts T0.5 which says that

$$
\int_{E} \sum_{i=1}^{\infty} E \underline{T}^{k} r=\int r
$$


To get (C) we will show

(2) $T\left(\sum_{i=1}^{\infty}\left(r_{i}+h_{i}\right)\right)=\sum_{i=1}^{\infty}\left(r_{i}+h_{i}\right)$ a.e. and we will then use T0.6 to show that $\sum_{i=1}^{\infty}\left(r_{i}+h_{i}\right)$ is a constant a.e.

We will get (2) from

(3) $T \sum_{i=1}^{n} r_{i}=\sum_{i=1}^{n} r_{i}-r_{1}+r_{n+1}+\sum_{i=1}^{n+1} l_{i}$.

(4) $T \sum_{i=1}^{n} h_{i}=\sum_{i=1}^{n} h_{i}-h_{1}+h_{n+1}+\sum_{i=1}^{n+1} l_{i}^{\prime}$.

Because of (B) $\lim \sup _{n \rightarrow \infty} r_{n}(x)=0$ a.e. (and $\lim \sup _{n \rightarrow \infty} h_{n}(x)=0$ a.e.) this shows that $\lim _{n \rightarrow \infty} T\left(\sum_{i=1}^{n}\left(r_{i}+h_{i}\right)\right)=\sum_{i=1}^{\infty}\left(r_{i}+h_{i}\right)$ a.e. and by the monotone convergence theorem we get (2).

(D) We can now show that

(1) $\left|\sum_{i=1}^{n} T^{i}(r-h) x\right|<B$, for all $n$ (a.e.) since for a.e. $x$,

$$
\sum_{i=1}^{n} T^{i} r(x)=\sum_{i=1}^{n} r_{i}(x)+\sum_{i=1}^{n}\left(\sum_{j=1}^{n-i} T^{j} l_{i}\right)(x)<B+\sum_{i=1}^{n} T^{i} h(x)
$$

and

$$
\sum_{i=1}^{n} T^{i} h(x)=\sum_{i=1}^{n} h_{i}(x)+\sum_{i=1}^{n}\left(\sum_{j=1}^{n-i} T^{j} l_{i}\right)(x)<B+\sum_{i=1}^{n} T^{i} r(x) .
$$

(E) It is easy to see that if $\left|\sum_{i=1}^{n} T^{i}(h-r)(x)\right|<B$ for all $n$, a.e. then the inequality must hold for all $x$. The singular part of $\sum_{i=1}^{\infty} T^{i} \delta_{0}$ is finite and the absolutely continuous part of $\sum_{i=1}^{n} T^{i} \delta_{0}$ convoluted with $h-r$ is continuous. This and L3 give (E).

(F) To see that $B$ is independent of $r$ and $h$ (for the support of $r$ and $h$ in a fixed $I$ and $0 \leqq r \leqq 1,0 \leqq h \leqq 1$ and $\left.\int h=\int r=1\right)$, we note that $B$ must be $<(4 / \alpha) \cdot K$ $\cdot|J|^{-1}(|J|=$ length of $J$ ) (equation (B)(5)).

We will now go from T2.1 and T2 to T1 by a series of easy lemmas.

L4. If $\int x^{2} u=\infty$ (and the walk is recurrent),

$$
\lim _{x \rightarrow \infty} h_{x}(A, B)=\lim _{x \rightarrow-\infty} h_{x}(A, B)
$$

( $A$ and $B$ are finite intervals).

Proof. L4 follows from the following: given $\varepsilon$ and $N$ there is $y>N$ such that (A) $h_{y}((-N, N),(-\infty,-N))<\varepsilon$ or $h_{-y}((-N, N),(N, \infty))<\varepsilon$. By results in [2, p. 575], one of the ladder random variables has infinite expectation. The classical renewal theorem then gives (A).

\{For the sake of a unified and self-contained treatment we will give a proof of (A) using only the classical renewal theorem (for $u$ with support on $(0, \infty)$ ). The existence of the limit in that theorem is implied by T1.1 and its identification as $1 / \int x u$ follows simply from the fact that $\int_{a}^{\infty} u * \psi_{(-\infty, a)}=\int x u$, and equation (2) below. $\}$

Before proving (A) we will give three definitions.

1. $l$ is the operator, multiplication by the function $l$.

2. $\alpha_{v}=\psi_{(-\infty, 0)} \sum_{i=0}^{\infty}\left(T \bar{\psi}_{(0, \infty)}\right)^{i}(v)$.

3. $\beta_{v}=\psi_{(0, \infty)} \sum_{i=0}^{\infty}\left(T \psi_{(0, \infty)}\right)^{i}(v)$. 
$\left(\int_{I} \beta_{v}<\infty\right.$ for any finite interval $I$ and the bound is independent of $v$ by L2.1.) We will sometimes let $v$ be a measure and sometimes the Radon-Nikodym derivative of an absolutely continuous measure. $\alpha_{v}$ and $\beta_{v}$ will then be measures or derivatives of measures respectively. $\alpha_{v}$ is the distribution of the position of the first hit to $(-\infty, 0)$ starting with the distribution $v$ while $\beta_{v}$ is the distribution of the expected number of hits in $(0, \infty)$ before hitting $(-\infty, 0)$.

(1) $\int-x \alpha_{\delta_{z}}=\infty$ for any $z>0$ implies (A). To see (1) let $\tilde{H}_{x}(A, B)$ be the distribution of $H_{x}(A, B)\left(\tilde{H}_{x}(A, B)=\psi_{A} \sum_{i=0}^{\infty}\left(\bar{\psi}_{B}^{\prime} T\right)^{i} \delta_{x}\right)$. Let $H_{x}^{\prime}(A, B)$ be the expected number of times we hit $A$ before $B$ starting at $x$ for the random walk given by $\alpha_{\delta_{z}} * \delta_{-z}$. The renewal theorem then gives $\lim _{y \rightarrow \infty} H_{y}^{\prime}((-N, N),(-\infty,-N))=0$. This implies that $\lim _{y \rightarrow \infty} H_{y}((-N, N),(-\infty,-N))=0$ because

$$
\tilde{H}_{y}((-N, N),(-\infty,-N))=\tilde{H}_{y}^{\prime}((-N, N),(-\infty,-N)) * \tilde{H}_{0}((-z, \infty),(-\infty,-z)) .
$$

$\lim _{y \rightarrow \infty} \tilde{H}_{y}^{\prime}((-N, N),(-\infty,-N))=0$ by (1) and the classical renewal theorem. $\int_{I} \tilde{H}_{0}((-z, \infty),(-\infty,-z))<\infty$ by L2.1 or the remark after the definition of $\beta_{v}$. ( $I$ is an arbitrary interval.)

(2) $\alpha_{v}=\psi_{(-\infty, 0)} T \beta_{v}$. This is easy to see.

(3) Let $r$ be the derivative of a probability measure with compact support, then $\lim _{\inf _{x \rightarrow \infty}} \beta_{r}(x)=\gamma>0$ implies $-\int-x \alpha_{r}=\infty$ (and this, because of T0.3 and (1), implies (A)). (3) is true because

$$
\int_{-\infty}^{0}-x\left(u * \psi_{(0, \infty)}\right)=\int_{-\infty}^{0} x^{2} u
$$

Let $r$ be the measure whose derivative is $(1 / 2 N) \psi_{(0,2 N)}$. Then

$$
\beta_{r}(x)=\left[\sum_{i=0}^{\infty}\left(T \bar{\psi}_{(0, \infty)}\right)^{i}(r)\right](x)=\int r \cdot \sum_{i=0}^{\infty}\left(\bar{\psi}_{(0, \infty)} \tilde{T}\right)^{i}\left(\delta_{x}\right) \text { for } x>0 .
$$

The first equality is simply the definition of $\beta_{r}$ for $x>0$ and the second comes from T0.2. Because of (3) we can assume $\lim \inf _{x \rightarrow \infty} \beta_{r}(x)=0$ but then the right side of (4) gives (A) for $\tilde{T}$ and hence for $T$.

L5. If the first part of $\mathrm{T} 1$ were false there would be $a \gamma>0$ and $x_{0}$ such that for any $N$ there would be integers $K$ and $L, K>N, L>0$ and

$$
\left|\int\left[f \cdot \sum_{i=1}^{n} T^{i}\left(T^{K} \delta_{x_{0}}\right)\right]-\int\left[f \cdot \sum_{i=1}^{n} T^{i}\left(T^{K+L} \delta_{x_{0}}\right)\right]\right|>\gamma
$$

for all $n$ large enough.

Proof. Using T0.2 we get that if T1 were false for $\tilde{T}$ there would be a $\gamma>0$ and arbitrarily large integers $K$ and $L$ such that

$$
\left|\int f \cdot \sum_{i=1}^{K} T^{i} \delta_{x_{0}}-\int f \cdot \sum_{i=1}^{K+L} T^{i} \delta_{x_{0}}\right|>2 \gamma
$$


fixing $K$ and $L$ and letting $u_{1}=T^{K} \delta_{x_{0}}$ and $u_{2}=T^{K+L} \delta_{x_{0}}$ we get

$$
\left|\int f \cdot \sum_{i=1}^{n+L} T^{i} u_{1}-\int f \cdot \sum_{i=1}^{n} T^{i} u_{2}\right|>2 \gamma \text { for all } n \text {. }
$$

For each interval $J$ we have

$$
\lim _{i \rightarrow \infty} \int_{J} T^{i} u_{1}=0
$$

(2) and (3) give L5.

L6. For any interval $I$ of length $1, \varepsilon>0, u_{1}=T^{K} \delta_{x_{0}}, u_{2}=T^{K+L} \delta_{x_{0}}$, we have: if $K$ is large enough then

$$
\left|\int f \cdot \sum_{j=1}^{n} T^{j} u_{i}-\int\left(f * \psi_{I}\right) \sum_{j=1}^{n} T^{j} u_{i}\right|<\varepsilon \text { for all } n, i=1,2 .
$$

Proof. T0.7 and T2 imply that there is an $M$ such that if $|t|<1$ and $|z|>M$ then

$$
\left|\int f \cdot \sum_{i=1}^{n} T^{i}\left(\delta_{z}-\delta_{z+t}\right)\right|<\frac{1}{2} \varepsilon .
$$

From (1) we immediately get

$$
\left|\int\left(f-f * \psi_{I}\right)\left(\sum_{i=1}^{n} T^{i} \delta_{z}\right)\right|<\frac{1}{2} \varepsilon \text { for all } n \text { and }|z|>M .
$$

L6 follows from (2) and T2 because if $K$ is large enough

$$
\int u_{i} \psi_{(-M, M)}<\frac{1}{2} \frac{\varepsilon}{B}
$$

where $B$ is the bound in $\mathrm{T} 2, i=1,2$.

L7. Let $J$ be a finite interval containing the support off. Let $h_{u_{1}}(J)$ be the distribution of the position of the first hit in $J$ starting with distribution $u_{1}$. Then given $\varepsilon$ there is an $N$ such that for $n>N$,

$$
\left|\int f\left(\sum_{i=1}^{n} T^{i} u_{1}\right)-\int f\left(\sum_{i=1}^{n} T^{i} h_{u_{1}}(J)\right)\right|<\varepsilon .
$$

Proof. Let $J \underline{T}$ be the transformation that agrees with $T$ on functions with support outside of $J$ and sends functions with support in $J$ into 0 .

Let $\alpha_{n}=\left({ }_{J} \underline{T}\right)^{n} u_{1}$

$$
h_{u_{1}}(J)=\sum_{i=1}^{\infty} \alpha_{i}
$$

$$
\begin{aligned}
f \cdot \sum_{i=1}^{n} T^{i} u_{1} & =f \sum_{i=1}^{n} \sum_{j=1}^{n-i} T^{j} \alpha_{i} \\
& =f \cdot \sum_{i=1}^{N} \sum_{j=1}^{n-i} T^{j} \alpha_{i}+f \cdot \sum_{j=1}^{n-N} T^{j}\left(\left[1-\psi_{j}\right](J \underline{T})^{N} u_{1}\right),
\end{aligned}
$$

where $N$ is an arbitrary integer to be determined later and $n>N$. 
If $N$ is large enough $\left|\left(1-\psi_{J}\right)(J \underline{T})^{N} u_{1}\right|$ can be made arbitrarily small and so by T2 we could choose $N$ so that

$$
\left|\int f \cdot \sum_{j=1}^{n-N} T^{j}\left(\left[1-\psi_{J}\right](J \underline{T})^{N} u_{1}\right)\right|<\frac{1}{2} \varepsilon \text { for all } n>N .
$$

For fixed $N$ we have

$$
\lim _{n \rightarrow \infty}\left|\int f \cdot \sum_{i=1}^{N} \sum_{j=1}^{n-i} T^{j} \alpha_{i}-\int f \sum_{j=1}^{n} T^{j}\left(\sum_{i=1}^{N} \alpha_{i}\right)\right|=0 .
$$

Putting (2), (3) and (4) together we get that for $N$ large enough

$$
\left|\int f \sum_{i=1}^{n} T^{i} u_{1}-\int f \cdot \sum_{i=1}^{n} T^{i}\left(\sum_{i=1}^{N} \alpha_{i}\right)\right|<\frac{3}{4} \varepsilon .
$$

Now use T2, (1) and (5) to get L7.

L8. Let $g$ be a continuous function with support in a finite interval $J$. Assume $\int g=0$ and $|g(x)| \leqq 1$. Then given $\varepsilon$ we can find a finite number of disjoint intervals $J_{i}$ such that $J=\bigcup_{i=1}^{P} J_{i}$ and if $x$ and $y$ are in the same $J_{i}$ then

$$
\left|\int g \cdot\left[\sum_{i=1}^{n} T^{i}\left(\delta_{x}-\delta_{y}\right)\right]\right|<\varepsilon \text { for all } n .
$$

Proof. T2 says that given $J$ there is a $B$ such that

$$
\left|\int f \cdot \sum_{i=0}^{m} T^{i} \delta_{x}\right|<B \cdot \sup |f| \text { for all } x \text { and } m
$$

and for all functions $f$ with support in $J$ and $\int f=0$.

Next notice that

$$
\int g \cdot\left[\sum_{i=1}^{n} T^{i}\left(\delta_{x}-\delta_{y}\right)\right]=\int\left(g-{ }_{t} g\right) \cdot \sum_{i=1}^{n} T^{i} \delta_{x}
$$

where ${ }_{t} g(x)=g(x-t)$ and $t=y-x$.

Pick an $\alpha>0$ such that if $|t|<\alpha$ then sup $\left|g-{ }_{t} g\right|<\varepsilon / B$. If the length of each $J$ is less than $\alpha$ then L8 will follow from (1) and (2).

L9. Let $u_{1}=T^{K} \delta_{x_{0}}$ and $u_{2}=T^{K+L} \delta_{x_{0}}$ and let $\bar{J}$ be a subinterval of $J$. Then given $\varepsilon$ there is an $N$ such that if $K($ and $K+L)>N$ then $\left|h_{u_{1}}(\bar{J}, J-\bar{J})-h_{u_{2}}(\bar{J}, J-\bar{J})\right|<\varepsilon$. $\left(h_{v}(A, B)\right.$ is the probability of hitting $A$ before $B$ starting with the distribution $\left.v.\right)$

Proof. Case 1. $\int x^{2} u=\infty$. By T2.1 and L4 there is an $M$ such that if $|z|>M$ and $|y|>M$ then

$$
\left|h_{z}(\bar{J}, J-\bar{J})-h_{y}(\bar{J}, J-\bar{J})\right|<\frac{1}{4} \varepsilon .
$$


If $N$ is large enough $\int u_{i} \psi_{(-M, M)}<\frac{1}{4} \varepsilon, i=1,2$ and so

$$
\left|h_{u_{i}}(\bar{J}, J-\bar{J})-\int_{|y|>M} u_{i} \cdot h_{y}(\bar{J}, J-\bar{J})\right|<\frac{1}{4} \varepsilon
$$

(note $h_{u_{i}}(\bar{J}, J-\bar{J})=\int_{-\infty}^{\infty} u_{i} h_{y}(\bar{J}, J-\bar{J})$ by T0.3). L9 follows from (1) and (2).

Case 2. $\int x^{2} u<\infty$. In this case use T2.1 to choose $M$ so that if $z$ and $y$ are both $>M$ or both $<-M$

$$
\left|h_{z}(\bar{J}, J-\bar{J})-h_{y}(\bar{J}, J-\bar{J})\right|<\frac{1}{4} \varepsilon .
$$

Now use the central limit theorem to show that if $K$ and $K+L$ are large enough

$$
\left|\frac{1}{2}-\int u_{i} \psi_{(M, \infty)}\right|<\frac{1}{4} \varepsilon \text { and }\left|\frac{1}{2}-\int u_{i} \psi_{(-\infty,-M)}\right|<\frac{1}{4} \varepsilon
$$

$i=1,2$. L7 follows immediately from (3) and (4).

Proof of (1) of T1. Assume the contrary. By L5,

$$
\left|\int f \cdot \sum_{i=1}^{n} T^{i}\left(T^{K} \delta_{x_{0}}\right)-\int f \cdot \sum_{i=1}^{n} T^{i} \cdot\left(T^{K+L} \delta_{x_{0}}\right)\right|>\gamma
$$

and by L6 if $K$ is large enough we get

$$
\left|\int\left(f * \psi_{I}\right) \sum_{i=1}^{n} T_{i}\left(T^{K} \delta_{x_{0}}\right)-\int\left(f * \psi_{I}\right) \sum_{i=1}^{n} T^{i}\left(T^{K+L} \delta_{x_{i}}\right)\right|>\frac{9}{10} \gamma .
$$

By L7 we get for $K$ sufficiently large and all $n$ large enough with respect to $K$

$$
\left|\int\left(f * \psi_{I}\right) \sum_{i=1}^{n} T^{i} h_{u_{1}}(J)-\int\left(f * \psi_{I}\right) \sum_{i=1}^{n} T^{i} h_{u_{2}}(J)\right|>\frac{8}{10} \gamma .
$$

Apply L8 to $g=f * \psi_{I}$ and $\varepsilon=\gamma / 10$ to get $J_{i}, i=1, \ldots, P$. Then use L9 to get (for $K$ large enough, and $B$ the bound in T2)

$$
\left|h_{u_{1}}\left(J_{i}, J-J_{i}\right)-h_{u_{2}}\left(J_{i}, J-J_{i}\right)\right|<\frac{\gamma}{10 P B}, \quad i=1, \ldots, P .
$$

This says that $h_{u_{1}}(J)=v+v^{\prime}$ where $|v|<\gamma / 10 B$ and $h_{u_{2}}(J)$ and $v^{\prime}$ give each $J_{i}$ the same measure.

By T2

$$
\left|\int\left(f * \psi_{I}\right) \sum_{i=1}^{n} T^{i} v\right|<\frac{1}{10} \gamma
$$

and by $\mathrm{L} 8$

$$
\left|\int\left(f * \psi_{I}\right) \sum_{i=1}^{n} T^{i} v^{\prime}-\int\left(f * \psi_{I}\right) \sum_{i=1}^{n} T^{i} h_{u_{2}}(J)\right|<\frac{1}{10} \gamma
$$

hence

$$
\left|\int\left(f * \psi_{I}\right) \sum_{i=1}^{n} T^{i} h_{u_{1}}(J)-\int\left(f * \psi_{I}\right) \sum_{i=1}^{n} T^{i} h_{u_{2}}(J)\right|<\frac{2}{10} \gamma \text { for all } n
$$

but this contradicts (1). 
Proof of (2) of T1. Assume this were false. There would be a $\gamma>0$ such that for each $N$ we could find $x$ and $y, x>N ; y>N$ for which

$$
\left|\int f \sum_{i=1}^{n} T^{i} \delta_{x}-\int f \sum_{i=1}^{n} T^{i} \delta_{y}\right|>\gamma
$$

for all $n$ large enough. By L6 if $N$ is large enough

and by $L 7$

$$
\left|\int\left(f * \psi_{I}\right) \sum_{i=1}^{n} T^{i} \delta_{x}-\int\left(f * \psi_{I}\right) \sum_{i=1}^{n} T^{i} \delta_{y}\right|>\frac{9}{10} \gamma
$$

$$
\left|\int\left(f * \psi_{I}\right) \sum_{i=1}^{n} T^{i} h_{x}(J)-\int\left(f * \psi_{I}\right) \sum_{i=1}^{n} T^{i} h_{y}(J)\right|>\frac{8}{10} \gamma
$$

for $N$ sufficiently large and all $n$ large enough with respect to $N$.

Now apply L8 with $g=f * \psi_{I}$ and $\gamma / 10$ to get $J_{i}, i=1, \ldots, P$. Then use T2.1 to get ( $N$ large enough)

$$
\left|h_{x}\left(J_{i}, J-J_{i}\right)-h_{y}\left(J-J_{i}\right)\right|<\frac{\gamma}{10 P B}, \quad i=1, \ldots, P .
$$

Use this as before to contradict (1).

THEOREM 3. In $\mathrm{T} 1$,

If $\int x^{2} u=\infty, \lim _{x \rightarrow \pm \infty} \bar{f}(x)=0$.

$$
\lim _{x \rightarrow \pm \infty} \bar{f}(x)= \pm \frac{\int x f}{\int x^{2} u} .
$$

Proof. Case 1. $\int x^{2} u=\infty$. It is easy to see that the proof of $\mathrm{T} 1$ gives us in this case

$$
\lim _{x \rightarrow \infty} \bar{f}(x)=\lim _{x \rightarrow-\infty} \bar{f}(x) .
$$

Since $\lim _{n \rightarrow \infty} \int f \cdot \sum_{i=1}^{n} T^{i} \delta_{0}$ exists we have: given $\varepsilon$ there is an $N$ such that

$$
\left|\int f \cdot \sum_{i=1}^{m} T^{i}\left(T^{N} \delta_{0}\right)\right|<\varepsilon \text { for } m=1,2, \ldots
$$

Since (2) is also true for $\tilde{T}$ we get by T0.2

$$
\left|\int\left(\sum_{i=1}^{m} T^{i}(f)\right)\left(\tilde{T}^{N} \delta_{0}\right)\right|<\varepsilon, \quad m=1,2, \ldots
$$

For each $K$ we can choose $N$ large enough so that $\int_{-K}^{K} \tilde{T}^{N} \delta_{0}<\varepsilon / B(B$ is the bound in T2) and applying this to (3) we get

$$
\left|\int_{|x|>K} \sum_{i=1}^{m} T^{i}(f)\left(\tilde{T}^{N} \delta_{0}\right)\right|<2 \varepsilon .
$$


This and T2 show that the limits in (1) must be 0 by the bounded convergence theorem.

Case 2. $\int x^{2} u<\infty$. In this case (2), (3) and (4) above are still true and (4) plus the central limit theorem gives

(1') $\lim _{x \rightarrow \infty} \bar{f}(x)=-\lim _{x \rightarrow-\infty} \bar{f}(x)=c$.

(2') $c$ only depends on $T$ and $\int x f$, i.e., if $\int x f=0$ then $\lim _{x \rightarrow \infty} \bar{f}(x)=0$.

We can see $\left(2^{\prime}\right)$ as follows:

Let $d(x)$ be the function $d(x)=1-x$ for $0 \leqq x<1, d(x)=1+x$ for $-1 \leqq x \leqq 0$, $d(x)=0$ for $|x|>1$. It will be enough to show that $\lim _{x \rightarrow \infty}|\bar{f} * d|(x)=0$. This follows by taking Fourier transforms and noting that

$$
\int \sum_{n=1}^{\infty}|\hat{f}||\hat{d}|\left|\hat{u}^{n}\right|<\infty
$$

because $\hat{d}$ is integrable and bounded,

$$
\sum_{i=1}^{n}\left|\hat{u}^{n}(\theta)\right|<\frac{\alpha}{\theta^{2}}
$$

for some $\alpha>0$ and all $\theta$ in some neighborhood of 0 and bounded on the rest of the line, and $|\hat{f}(\theta)|<\beta \theta^{2}$ for some $\beta>0$ and all $\theta$ in some neighborhood of 0 .

Because of $\left(2^{\prime}\right)$ we can use any $f$ we want to evaluate $c$.

Let ${ }_{\alpha} d(x)=d(x-\alpha)$. Then $\left(1^{\prime}\right)$ says that

(3') $\lim _{x \rightarrow \infty} \lim _{n \rightarrow \infty} \int .\left({ }_{x} d-{ }_{x+1} d\right) \sum_{i=1}^{n} T^{i} \delta_{0}=-\lim _{x \rightarrow-\infty} \lim _{n \rightarrow \infty} \int\left({ }_{x} d-{ }_{x+1} d\right) \sum_{i=1}^{n} T^{i} \delta_{0}$.

Our theorem will follow immediately from $\left(3^{\prime}\right)$ and the following (essentially p. 28.4 of Spitzer [6]):

$$
\lim _{x \rightarrow \infty} \frac{1}{x} \lim _{n \rightarrow \infty} \int\left(2 d-{ }_{x} d-{ }_{-x} d\right) \sum_{i=1}^{n} T^{i} \delta_{0}=2\left(\int x^{2} u\right)^{-1} .
$$

$\left(4^{\prime}\right)$ is equivalent to

$$
\lim _{x \rightarrow \infty} \frac{1}{x} \lim _{n \rightarrow \infty} \int(1-\cos x \theta) \hat{d} \sum_{i=1}^{n} \hat{u}^{n}=2 \int x^{2} u .
$$

This can be evaluated as in Spitzer.

THEOREM 4. Let $v^{\prime \prime}$ be the absolutely continuous part of the measure v. Let

$$
C_{n}=\int_{0}^{1} \sum_{i=1}^{n} T^{i} \delta_{0} \text { and } l_{n}(x)=\left(\sum_{i=1}^{n} T^{i} \delta_{0}\right)^{\prime \prime}-C_{n} .
$$

Then there is a function $l_{\infty}(x)$ such that $\int_{a}^{b}\left|l_{\infty}(x)\right|<\infty$ for all $a, b$ and $l_{n}(x)$ converges to $l_{\infty}(x)$ in the $L_{1}$ sense on any finite interval. 
Proof. Let

$$
C_{n, v}=\int_{0}^{1} \sum_{i=1}^{n} T^{i}(v) \text { and } l_{n, v}=C_{n, v}-\left(\sum_{i=1}^{n} T^{i} v\right)^{\prime \prime}
$$

where $v$ is a probability measure with bounded support.

(1) Let $v$ be an absolutely continuous measure with bounded support and bounded derivative $f$. Then $l_{n, v}$ converges to some $l_{\infty, v}$ in the $L_{1}$ sense on any finite interval. To see (1): let ${ }_{x} f(y)=f(y-x)={ }_{x} f$. T1 implies

$$
\left[\sum_{i=1}^{\infty} T^{i}\left(f * \psi_{(0,1)}-{ }_{x} f\right)\right](y)
$$

converges for all $x$ and $y$ and T2 implies that given $K$ there is a $B$ such that

for all $|x|<K$ and all $y$.

$$
\left|\left[\sum_{i=1}^{n} T^{i}\left(f * \psi_{(0,1)}-{ }_{x} f\right)\right] y\right|<B
$$

$$
\begin{aligned}
{\left[\sum_{i=1}^{n} T^{i}\left(f * \psi_{(0,1)}-{ }_{x} f\right)\right](0)=\int \psi_{(0,1)} \sum_{i=1}^{n} T^{i}(f)-\left[\sum_{i=1}^{n} T^{i} f\right](-x) } & =l_{n, f}(-x) \\
& =l_{n, v}(-x)
\end{aligned}
$$

This shows (1).

(2) Given an integer $K$ there is a $B$ such that $\int_{-K}^{K}\left|l_{n, v}\right|<B$ for all probability measures $v$ and all $n$. To see (2): By T2 and T1, there is a $B_{1}$ such that

(a) $\left|\int_{-K}^{K} l_{n, v}\right|<B_{1}$ for all $n$ and $v$.

(b) $\left|\int g l_{n, v}\right|<B_{1}$ for all $|g(x)| \leqq 1, \int g=0$ and support of $g$ is in $(-K, K)$. If $\int_{-K}^{K}\left|l_{n, v}\right|>4 B$, we could find 2 sets, $R$ and $S$ in $-K, K$ of equal measure, such that if we let $g=\psi_{R}-\psi_{S}$ then (b) will be violated.

T4 follows immediately from (1) and (2) and the fact that given $\varepsilon$ there is an $N$ such that $T^{N} \delta_{0}$ can be written as $v_{1}+v_{2}$ where $v_{1}$ and $v_{2}$ are positive measures $\int v_{1}<\varepsilon$ and $v_{2}$ is absolutely continuous with bounded support and bounded derivative. This shows that $l_{n}$ forms a Cauchy sequence in $L_{1}$ on any finite interval.

L10. Let $v$ be a probability measure with bounded support then

$$
\lim _{b \rightarrow \infty} \lim _{n \rightarrow \infty} \int\left(\psi_{(b-1 / 2, b+1 / 2)}+\psi_{(-b-1 / 2,-b+1 / 2)}-2 \psi_{(-1 / 2,1 / 2)}\right) \sum_{i=1}^{n} T^{i} v=-\infty .
$$

Proof. The proof will be very similar to that of $\mathrm{T} 2$.

We will start by letting $r=\psi_{(b, b+1)}$ and $h=\psi_{(-1 / 2,1 / 2)}$. Then, just as in the proof of T2 we will define $r_{i}$ and $l_{i}$. Let $I=(-1 / 2,1 / 2)$ and let $I+b=(b-1 / 2, b+1 / 2)$.

(1) For a fixed interval $I, \int_{I+b} \sum_{i=1}^{\infty} r_{i}$ can be made as large as we want by making $b$ large enough (recall that $r$ depends on $b$ ). To see (1) note that for a given large number $L$ we can choose $v$ so that $\int_{I+b} \sum_{i=1}^{n} T^{i} r>L$ for all $b$ by T0.4 (the integral does not depend on $b$ ). Next given $\varepsilon$ we can choose $b$ so large that

$$
\int_{-1 / 2}^{1 / 2} \sum_{i=1}^{n} T^{i} r<\frac{\varepsilon}{n}
$$


This last inequality implies that $\int \sum_{i=1}^{n} l_{i}<\varepsilon / n$ and this in turn implies that $\int\left[\left(T^{i} r\right)-r_{i}\right]<\varepsilon / n$ for $1 \leqq i \leqq n$. From this we get that $\int_{I+b} \sum_{i=1}^{n} r_{i}>L-\varepsilon$ which is what we want.

(2) For fixed $C, \int_{-C}^{C} \sum_{i=1}^{\infty} r_{i}$ is bounded independently of $b$. To see this we just repeat the argument in $B$ of T3.2. We get (4) in that argument as before namely for each fixed $K$ and every $n$ there is a set $S_{n}^{\prime}$ of nonzero measure such that

$$
T^{K}\left(\sum_{i=1}^{n} r_{i}\right)(x)<K \text { for } x \in S_{n}^{\prime} .
$$

Note that $S_{n}^{\prime}$ was included in the support of $h$ which in this case is $\left(-\frac{1}{2}, \frac{1}{2}\right)$. Next we find a $K$ and $\alpha>0$ such that $\left(T^{K} \delta_{0}\right)^{\prime \prime}(x)>\alpha$ for all $|x|<2 C$. We now have that

$$
\int_{-C}^{C} \sum_{i=1}^{n} r_{i}<\frac{2 K}{\alpha}
$$

because otherwise $T^{K}\left(\sum_{i=1}^{n} r_{i}\right)(x)>2 K$ for all $|x|<\frac{1}{2}$ (and hence some $x$ in $S_{n}^{\prime}$ ).

(1) and (2) imply

(3) $\int \sum_{i=1}^{\infty} r_{i}\left(-\psi_{I}+\psi_{I+b}\right)$ tends to $\infty$ as $b$ tends to $\infty$.

$$
\sum_{i=1}^{n} T^{i}(r-h)=\sum_{i=1}^{n} r_{i}+\sum_{i=1}^{n} \sum_{j=1}^{n-i} T^{j} l_{i}-\sum_{i=1}^{n} T^{i} h .
$$

If we can show

$$
\left|\int\left(\sum_{i=1}^{n} \sum_{j=1}^{n-i} T^{j} l_{i}-\sum_{i=1}^{n} T^{i} h\right) \cdot\left(\psi_{I}-\psi_{I+b}\right)\right|<1
$$

for each fixed $b$ and large enough $n$, then (3), (4) and (5) will imply L10 for the case when $\psi_{I}$ is the derivative of $v$.

To see (5) note that $\sum_{i=1}^{\infty} l_{i}=h$ by equation (C)(1) of T3.2. Pick $K$ so that

$$
\int\left|\sum_{i=1}^{K} l_{i}-h\right|<\varepsilon
$$

where $\varepsilon$ is chosen so that if $B$ is the bound for $\left|\sum_{i=1}^{m} T^{i} \delta_{x}\left(\psi_{I}-\psi_{I+b}\right)\right|$ given by T3.2 ( $B$ may depend on $b$ but not on $x$ ) then $\varepsilon B<\frac{1}{2}$. Now rewrite the left side of (5).

$$
\begin{aligned}
\mid \int\left(\sum_{i=1}^{K} \sum_{j=1}^{n-i} T^{j} l_{i}\right. & \left.-\sum_{i=1}^{K} \sum_{j=1}^{n} T^{j} l_{i}\right)\left(\psi_{I}-\psi_{I+b}\right) \\
& +\int\left(\sum_{i=K+1}^{n} \sum_{j=1}^{n-i} T^{i} l_{i}-\sum_{i=K+1}^{\infty} \sum_{j=1}^{n} T^{j} l_{i}\right)\left(\psi_{I}-\psi_{I+b}\right) \mid
\end{aligned}
$$

The first term of (6) tends to 0 as $n \rightarrow \infty$ because each $\int T^{n} l_{i} \cdot\left(\psi_{I}+\psi_{I+b}\right)$ tends to 0 . $K$ was chosen so that $\int\left|\sum_{i=K+1}^{\infty} l_{i}\right|<\varepsilon$ and because of the way we choose $\varepsilon$ and T0.3 the second term of $(6)$ is less than $\frac{1}{2}$. 
We have now shown L10 for the case when the derivative of $v$ is $\psi_{I}$. However for each interval $J$ we can use T2 to find a $\bar{B}$ depending only on $J$ and $I$ (not on $b$ ) such that

$$
\left|\int \sum_{i=1}^{n} T^{i}\left(\delta_{x}-\delta_{y}\right)\left(\psi_{I-b}+\psi_{I+b}-2 \psi_{I}\right)\right|<\bar{B}
$$

for $x$ and $y$ in $J$.

We can now use (7) and T0.3 to get L10.

THEOREM 5. Let $l_{\infty}(x)$ be the limit in T4. Then $\lim _{x \rightarrow \pm \infty} \int_{x}^{x+1} l_{\infty}(x)$ both exist. Either both limits are $-\infty$ or one is $-\infty$ and the other finite.

Proof. We will start with some definitions. If $J$ is an interval let ${ }_{a} J$ be the interval $J$ translated by $a$. If $v$ is a probability measure let

We can assume

$$
L_{v}\left(a J-{ }_{b} J\right)=\lim _{n \rightarrow \infty} \int\left(\psi_{a J}-\psi_{b J}\right) \sum_{i=1}^{n} T^{i} v
$$

(1) $\lim _{|x| \rightarrow \infty} L_{\delta_{x}}\left({ }_{a} J-{ }_{b} J\right)=0$ for each fixed $J, a$ and $b$, because otherwise we could easily see from T3 that both limits in T5 equal $-\infty$.

(2) Given $\varepsilon$ there exists an $N$ such that if $a>N$ and $|x|<1$ and $|y|<1$ then

$$
\lim _{n \rightarrow \infty}\left|\int \psi_{a^{J}} \sum_{i=1}^{n} T^{i} \delta_{x}-\int \psi_{a^{J}} \sum_{i=1}^{n} T^{i} \delta_{y}\right|<\varepsilon .
$$

To see (2): Fix $x-y$. We can then get an $N$ satisfying (2) by applying (1) with $b=a+x-y$. From this we get that there must be a set $E$ of nonzero measure such that (2) is true with the additional condition that $x-y \in E$. It is easy to see that (2) must also be true for $x$ and $y$ such that $x-y=x_{1}-y_{1}-\left(x_{2}-y_{2}\right)$ where $x_{1}-y_{1} \in E$ and $x_{2}-y_{2} \in E$. But the difference set of $E$ must contain an interval. Now cover $(-1,1)$ by a finite number of translates of this interval and write $x-y$ as one of a fixed finite number of numbers plus a number in this interval and we are finished.

(3) There is a $B$ such that if $|x-y|<1, J$ an interval of length less than 1 then

$$
\left|\int \psi_{J} \sum_{i=1}^{n} T^{i} \delta_{x}-\int \psi_{J} \sum_{i=1}^{n} T^{i} \delta_{y}\right|<B
$$

for all $n$. (3) follows easily from T2.

$$
\int\left(\psi_{(-1,1)}-\psi_{(x, x+2)}\right) \sum_{i=1}^{n} T^{i} \delta_{0}>K
$$

for all $x$ and $n$ where $K$ is a possibly negative number. To see (4): Let $v$ be the distribution of the first hit in $(x, x+2)$ starting at 0 . Then

$$
\int \psi_{(x, x+2)} \sum_{i=1}^{n} T^{i} \delta_{0}<\int \psi_{(x, x+2)} \sum_{i=1}^{n} T^{i} v=\int \psi_{(-1,1)} \sum_{i=1}^{n} T_{(-x-1)}^{i} v
$$


$\left({ }_{a} v=v\right.$ translated by $\left.a\right)$ and by (3) we get that

$$
\left|\int \psi_{(-1,1)} \sum_{i=1}^{n} T_{(-x-1)}^{i} v-\int \psi_{(-1,1)} \sum_{i=1}^{n} T^{i} \delta_{0}\right|<B
$$

for all $n$ and $v$ with support in $(-1,1)$. (4) says that

$$
\sup _{x} \int_{x}^{x+1} l_{\infty}(y) d y<\infty .
$$

If T5 were false we could see with the help of (1) that there would be numbers $M_{1}>M_{2}>M_{3}>M_{4}$ such that

$$
M_{1}>L_{\delta_{0}}(c I-I)>M_{2} \text { and } M_{3}>L_{\delta_{0}}(t I-I)>M_{4}
$$

for arbitrarily large $b$ and $c\left(I=\left(-\frac{1}{2}, \frac{1}{2}\right)\right)$.

(6) Because of (2), if $b$ and $c$ are large enough (5) will remain true if we replace $\delta$ by any probability measure on $I$.

Next choose a point $z,|z|$ very large (to be determined later) and $\varepsilon<\left(M_{2}-M_{3}{ }^{\prime} / 10\right.$ and define the measures $\alpha, \beta, \gamma$ to be the part of the distribution of the first hit in $I \cup{ }_{b} I \cup{ }_{c} I$ that lies in $I,{ }_{b} I,{ }_{c} I$ respectively (starting at $z$ ).

(7) $L_{\delta_{z}}(I-I)=L_{\alpha}\left({ }_{c} I-I\right)+L_{\beta}\left(b_{b} I-I\right)+L_{\beta}\left({ }_{c} I-{ }_{b} I\right)+L_{\gamma}\left({ }_{c} I-I\right)$. This follows from L7 (or from T2 and the fact that $\lim _{n \rightarrow \infty} \int_{J} T^{n} \delta_{x}=0$ for all $x$ and bounded $J$ ).

(8) By choosing $b$ and $c$ large we can make $|\beta|$ and $|\gamma|$ arbitrarily small. To see (8): Let us restrict our attention to $I$ and ${ }_{c} I$. Let $v_{1}, v_{2}$ be part of the distribution of the first hit in $I \cup{ }_{c} I$ starting at $z$ that lies in $I,{ }_{c} I$ respectively.

By (5) $\left|L_{\delta_{0}}\left(C_{c} I-I\right)\right|<M_{1}$ and so by $L 10, L_{\delta_{c}}(c I-I)$ can be made arbitrarily large by making $c$ large enough.

$$
\lim _{|z| \rightarrow \infty}\left|L_{v_{1}}(c I-I)-L_{v_{2}}(c I-I)\right|=0,
$$

by (1) and L7. We can now see, with the help of (3) that $\left|v_{2}\right|$ must be very small. $|\gamma|<\left|v_{2}\right|$. This and a similar argument for $\beta$ give (8).

(9) $L_{\alpha}\left({ }_{b} I-I\right)+L_{\beta}\left({ }_{b} I-I\right)+L_{\gamma}\left({ }_{b} I-I\right)$ tend to 0 as $|z| \rightarrow \infty$ and if $c$ is chosen large compared to $b$ we get by $(1),\left|L_{\alpha}\left({ }_{b} I-I\right)+L_{\beta}\left({ }_{b} I-I\right)\right|<\varepsilon$.

Using (9) and the fact that for $|z|$ large enough, $\left|L_{\delta_{z}}(c I-I)\right|<\varepsilon$ we can rewrite (7) as:

$$
\begin{aligned}
\mid L_{\alpha}\left({ }_{c} I-I\right)-L_{\alpha}\left({ }_{b} I-I\right)+ & L_{\beta}\left({ }_{c} I-{ }_{b} I\right)+L_{\gamma}\left({ }_{c} I-I\right) \mid \\
& =\left|L_{\alpha}\left({ }_{c} I-{ }_{b} I\right)+L_{\beta}\left({ }_{c} I-{ }_{b} I\right)+L_{\gamma}\left({ }_{c} I-I\right)\right|<2 \varepsilon .
\end{aligned}
$$

(11) By (5) and (6) we have $L_{\alpha}\left({ }_{c} I-{ }_{b} I\right)>M_{2}-M_{3}$.

(12) If $c$ is chosen large enough with respect to $b$ and $|\beta|$ forced to be small enough we have $\left|L_{\beta}\left({ }_{c} I-{ }_{b} I\right)\right|<\varepsilon$. To see (12):

$$
\left|L_{\delta_{b}}\left(C_{C} I-{ }_{b} I\right)-L_{\beta(1 /|\beta|)}\left({ }_{C} I-{ }_{b} I\right)\right|<B \text { by (3). }
$$


$L_{\delta_{b}}\left(c_{c} I-{ }_{b} I\right)=L_{\delta_{0}}\left({ }_{c-b} I-I\right)$ if $c$ is large compared to $b$ this last term is almost equal to $L_{\delta_{0}}\left({ }_{c} I-I\right)$ by $(1)$.

(13) By forcing $|\gamma|$ to be small enough we can insure that $L_{\gamma}\left({ }_{c} I-I\right)>-\varepsilon$. This comes from (4).

(10) together with (11), (12), and (13) gives a contradiction.

\section{Section 4}

THEOREM 1. A random walk on the line is transient if and only if

$$
\int_{-a}^{a} \operatorname{Re}\left(\frac{1}{1-\hat{u}(\theta)}\right) d \theta<\infty \text { for some } a \neq 0 .
$$

( $u$ is a probability measure equal to the distribution of the random variables of the random walk, and $\hat{u}(\theta)$ is its Fourier transform.)

(The proof will depend only on the proof of T3.2.)

L1. If Theorem 1 is true for all random walks such that $u$ has an absolutely continuous component then Theorem 1 is true for all random walks.

Proof. By the Chung-Fuchs theorem, a random walk is transient iff, for some $a$,

$$
\lim _{t \rightarrow 1} \int_{-a}^{a} \operatorname{Re}\left(\frac{1}{1-t \hat{u}(\theta)}\right) d \theta<\infty \quad(0<t<1) .
$$

Therefore we need only show that

$$
\lim _{t \rightarrow 1} \int_{-a}^{a}\left(\frac{1}{1-t \hat{u}(\theta)}\right) d \theta<\infty \text { for some } a
$$

iff

$$
\int_{-b}^{b} \operatorname{Re}\left(\frac{1}{1-\hat{u}(\theta)}\right) d \theta<\infty \quad \text { for some } b
$$

Now the hypothesis of $\mathrm{L} 1$ says that the above implication is true if $u$ has an absolutely continuous component. If $u$ does not have an absolutely continuous component we will change it a little so that it has one and show that this change will not affect either side of (1).

(2) We can always find a probability measure $m$ with an absolutely continuous component such that $\int_{-\infty}^{\infty} x^{i}(m-u) d x=0, i=0,1,2,3$ and $\int_{-\infty}^{\infty} x^{4}(m-u) d x<\infty$. (Assuming the support of $u$ is more than 2 points.)

If the moments of $u$ are not all finite then pick $N$ large enough so that at least 2 points of the support of $u$ are in $(-N, N)$. Let $m$ agree with $u$ outside $(-N, N)$ let $v=u \cdot \psi_{(-N, N)} / \int u \cdot \psi_{(-N, N)} \cdot v$ is a probability measure whose moments are all finite and we must find a probability measure $m^{\prime}$ with the same first 3 moments, finite 4th moment and an absolutely continuous component.

For the sake of notation we might as well assume that the moments of $u$ are finite and find $m$ as before. 
If the support of $u$ is more than 2 points then $\int x^{2} u>\left(\int x u\right)^{2}$. Now for any 2 numbers $a>b^{2}$ there is a canonical measure $v_{a, b}$ that gives measure $\frac{1}{2}$ to 2 points equidistant from $b$ and such that $\int x^{2} v_{a, b}=a$ (and $\int x v_{a, b}=b, \int v_{a, b}=1$ ). Next pick any measure $\bar{v}$ with an absolutely continuous component, and finite moments. Let ${ }_{y} \bar{v}$ be $\bar{v}$ translated by $y$. Consider the measure $\alpha t_{y} \bar{v}+\alpha(1-t)_{-y} \bar{v}=s_{y, \alpha, t}$. For each $N$ and $\varepsilon>0$ we can pick $y$ large enough, and $\alpha<\varepsilon$ so that

$$
\int x^{3} s_{y, \alpha, 1}>N, \quad \int x^{3} s_{y, \alpha, 0}<-N
$$

and

$$
\int s_{y, \alpha, l}, \quad\left|\int x s_{y, \alpha, t}\right| \text { and } \int x^{2} s_{y, \alpha, t}
$$

are all $<\varepsilon$ for all $0 \leqq t \leqq 1$. To each $s_{y, \alpha, t}$ add $c v_{a, b}$ (defined above). Choose $a, b, c$ so that if we call $m_{y, \alpha, t}=s_{y, \alpha, t}+(1-\alpha) v_{a, b}$ we have that

$$
\int x m_{y, \alpha, t}=\int x u, \quad \int x^{2} m_{y, \alpha, t}=\int x^{2} u \text { and } \int m_{y, \alpha, t}=1 .
$$

This determines $a, b$ and $c$ uniquely in terms of $y, \alpha$ and $t$ if $\varepsilon$ is small enough. (See the first two sentences in the paragraph.) Next if $N$ is large enough

$$
\int x^{3} m_{y, \alpha, 1}>\left|\int x^{3} u\right| \text { and } \int x^{3} m_{y, \alpha, 0}<-\left|\int x^{3} u\right| .
$$

$\left(\left|\int x^{3} c v_{a, b}\right|\right)$ are uniformly bounded for all of our choices of $\left.c v_{a, b}\right)$. By continuity $\int x^{3} m_{y, \alpha, l}=\int x^{3} u$ for some choice of $t$ between 0 and 1 .

$$
\frac{1}{1-t \hat{u}}-\frac{1}{1-t \hat{m}}=\frac{t(\hat{u}-\hat{m})}{(1-t \hat{u})(1-t \hat{m})} \text {. }
$$

There is a constant $C$ such that in some neighborhood of 0 ,

$$
\left|\frac{t(\hat{u}-\hat{m})}{(1-t \hat{u})(1-t \hat{m})}\right|<C \text { for all } t, 0 \leqq t \leqq 1,
$$

since for any probability measure $v$ there is a constant $K$ such that $1-|\hat{v}(\theta)|>K \theta^{2}$ in some neighborhood of 0 .

L2. If there is an a such that

$$
\lim _{t \rightarrow 1} \int_{-a}^{a} \operatorname{Re}\left(\frac{1}{1-t \hat{u}(\theta)}\right) d \theta<\infty
$$

then $\int_{-a}^{a} \operatorname{Re}(1 /(1-\hat{u}(\theta))) d \theta<\infty$.

Proof. This follows from the fact that

$$
\operatorname{Re}\left(\frac{1}{1-t \hat{u}(\theta)}\right) \geqq 0 \text { for } 0 \leqq t \leqq 1 .
$$


L3. If $u$ has an absolutely continuous component and if

$$
\int_{-a}^{a} \operatorname{Re}\left(\frac{1}{1-\hat{u}(\theta)}\right) d \theta<\infty \text { for some } a>0
$$

then

$$
\int_{-b}^{b} \operatorname{Re}\left(\frac{1}{1-\hat{u}(\theta)}\right) d \theta<\infty \text { for any } b>0
$$

Proof. This is easy and will be omitted.

L4. Let $f(x)$ be a real valued function with the following properties:

$$
\int|f(x)| \leqq 1, \quad \int f(x)=\int x f(x)=0, \quad \int x^{2} f(x)<\infty .
$$

$f$ is symmetric and $\hat{f}$ has support in $(-a, a)$. Then if

$$
\int_{-a}^{a} \operatorname{Re}\left(\frac{1}{1-\hat{u}(\theta)}\right) d \theta=K<\infty \text { for some } a \neq 0
$$

there is $a B>0$ and independent of $f$ such that

( $B$ will depend on $a$ and on $K$.)

$$
\lim _{n \rightarrow \infty} \int f \cdot \sum_{i=1}^{n} u^{(i)}<B
$$

Proof. $\hat{f}(\theta)$ must have the following properties. $|\hat{f}(\theta)| \leqq 1, \hat{f}(0)=0, \hat{f}^{\prime}(0)=0$, $\hat{f}^{\prime \prime}(0)<\infty, \hat{f}(\theta)$ is real and $\hat{f}(\theta)$ has compact support. From this we see that

(1) $\left|\hat{f}(\theta) \sum_{i=1}^{n} \hat{u}^{i}(\theta)\right|<C$ for all $\theta$ and all $n$. (We use here that $\hat{f}(\theta)<C^{\prime} \theta^{2}$ in some neighborhood of 0 , that $1-|\hat{u}(\theta)|>C^{\prime \prime} \theta^{2}$ in some neighborhood of 0 , that $\hat{u}(\theta)$ is continuous and $\hat{u}(\theta) \neq 1$ for $\theta \neq 0$.) From (1) we get

$$
\lim _{n \rightarrow \infty} \int_{-\infty}^{\infty} \hat{f}(\theta) \sum_{i=1}^{n} \hat{u}^{i}(\theta) d \theta=\int_{-\infty}^{\infty} \hat{f}(\theta) \frac{1}{1-\hat{u}(\theta)} d \theta .
$$

The right side of (2) is less than

$$
\int_{-a}^{a}\left|\operatorname{Re}\left(\frac{1}{1-\hat{u}(\theta)}\right)\right| d \theta
$$

where $(-a, a)$ includes the support of $\hat{f}$ and the left side of $(2)$ is $\lim _{n \rightarrow \infty} \int f \cdot \sum_{i=1}^{n} u^{(i)}$.

The next lemma will be an elaboration of L3.10.

L5. If $u$ has an absolutely continuous component and is recurrent then we can construct an $f$ satisfying the condition of $\mathrm{L} 4$ but with $\lim _{n \rightarrow \infty} \int f \sum_{i=1}^{n} u^{(i)}$ as large as we want.

Proof. Let $k$ be any function such that $k(x) \geqq 0, \int k(x) d x=1 . k(x)$ is symmetric $(\hat{k}(\theta)$ real) and $\hat{k}(\theta)$ has support in $(-a, a)$. Let $g(x)$ be the function

$$
\begin{aligned}
& g(x)=1, b-\frac{1}{2} \leqq x \leqq b+\frac{1}{2} \\
& g(x)=1,-b-\frac{1}{2} \leqq x \leqq-b+\frac{1}{2},
\end{aligned}
$$




$$
\begin{aligned}
& g(x)=2,-\frac{1}{2} \leqq x \leqq \frac{1}{2}\left(\text { call }\left(-\frac{1}{2}, \frac{1}{2}\right), I\right), \\
& g(x)=0, \text { all other } x,
\end{aligned}
$$

$b$ will be a very large number to be determined later. $f$ will equal $g * k * \psi_{I}$. We will start by examining some of the properties of $g$.

Let $g_{1}=\psi_{(b-1 / 2, b+1 / 2)}-\psi_{(-1 / 2,1 / 2)}$ then $g=g_{1}-{ }_{-b} g_{1}\left({ }_{-a} g_{1}\right.$ is $g_{1}$ translated by $\left.-a\right)$. We will restrict attention to $g_{1}$ for a while.

Let $r=\psi_{(b-1 / 2, b+1 / 2)}$ and $h=\psi_{(-1 / 2,1 / 2)}$. Let $T$ be the operator convolution by $u$. As in the proof of T3.2 we will define $r_{i}$ and $l_{i}$ as follows:

Let $r_{1}=r$ and $l_{1}=0$.

Let $r_{2}=T r_{1}-\inf \left(T r_{1}, h\right), l_{2}=\inf \left(T r_{1}, h\right)$.

Let $r_{3}=T r_{2}-\inf \left(T r_{2}, h-l_{2}\right), l_{3}=\inf \left(T r_{2}, h-l_{2}\right)$.

Let

$$
\begin{aligned}
& r_{i}=T r_{i-1}-\inf \left(T r_{i-1}, h-\sum_{j=1}^{i-1} l_{j}\right), \\
& l_{i}=\inf \left(T r_{i-1}, h-\sum_{j=1}^{i-1} l_{j}\right) .
\end{aligned}
$$

(1) For a fixed interval $I, \int_{I+b} \sum_{i=1}^{\infty} r_{i}$ can be made as large as we want by making $b$ large enough (recall that $r$ depends on $b$, and that $I+b$ is $I$ translated by $b$ ). To see (1) note that for a given large number $L$ we can choose $v$ so that

$$
\int_{I+b} \sum_{i=1}^{n} T^{i} r>L \text { for all } b
$$

by T0.4 (the integral does not depend on $b$ ). Next given $\varepsilon$ we can choose $b$ so large that

$$
\int_{-1 / 2}^{1 / 2} \sum_{i=1}^{n} T^{i} r<\frac{\varepsilon}{n}
$$

This last inequality implies that

$$
\int \sum_{i=1}^{n} l_{i}<\frac{\varepsilon}{n}
$$

and this in turn implies that $\int T^{i} r-r_{i}<\varepsilon / n$ for $1 \leqq i \leqq n$. From this we get that

$$
\int_{I+b} \sum_{i=1}^{n} r_{i}>L
$$

which is what we want.

(2) For fixed $C, \int_{-C}^{c} \sum_{i=1}^{\infty} r_{i}$ is bounded independently of $b$. To see this we just repeat the argument in (B) of T3.2. We get (4) in that argument as before, namely, for each fixed $K$ and every $n$ there is a set $S_{n}^{\prime}$ of nonzero measure such that

$$
T^{K}\left(\sum_{i=1}^{n} r_{i}\right)(x)<K \text { for } x \in S_{n}^{\prime} .
$$


Note that $S_{n}^{\prime}$ was included in the support of $h$ which in this case is $\left(-\frac{1}{2}, \frac{1}{2}\right)$. Next we find a $K$ and $\alpha>0$ such that $\left(T^{K} \delta_{0}\right)^{\prime \prime}(x)<\alpha$ for all $|x|<2 C$. We now have that

$$
\int_{-C}^{C} \sum_{i=1}^{n} r_{i}<\frac{2 K}{\alpha}
$$

because otherwise $T^{K}\left(\sum_{i=1}^{n} r_{i}\right)(x)>2 K$ for all $|x|<\frac{1}{2}$ (and hence some $x$ in $S_{n}^{\prime}$ ).

(3) Fix an interval $J$; then for any two intervals $I$ and $I^{\prime}$ of the same length and both included in $J$ we have that

$$
\left|\int_{I+b} \sum_{i=1}^{\infty} r_{i}-\int_{I^{\prime}+b} \sum_{i=1}^{\infty} r_{i}\right|
$$

is bounded independently of $I, I^{\prime}$ and $b$. (The bound may depend on $J$.) To see this define $h_{i}$ and $l_{i}^{\prime}$ as in T3.2, (C). Then $\sum_{i=1}^{\infty} h_{i}+r_{i}$ is a constant a.e. Apply (2) to the $h_{i}$ to get $\int_{J+b} \sum_{i=1}^{\infty} h_{i}$ is bounded independently of $b$. This implies (3).

$$
\int_{b-1 / 2}^{b+1 / 2} \sum_{i=1}^{\infty} r_{i}>\int_{x-1 / 2}^{x+1 / 2} \sum_{i=1}^{\infty} r_{i}+D
$$

where $D$ is a constant independent of $b$ and $x$. This comes from the fact that $\sum_{i=1}^{\infty} r_{i}+\sum_{i=1}^{\infty} h_{i}$ is a constant and that

$$
\int_{b-1 / 2}^{b+1 / 2} \sum_{i=1}^{\infty} h_{i}<D
$$

where $D$ is independent of $b$.

$$
\int\left(\psi_{I}\right) *\left(\sum_{i=1}^{\infty} r_{i}\right) \cdot\left(-k+{ }_{b} k\right)
$$

tends to $+\infty$ as $b$ tends to $+\infty$. To see this divide the integral into three parts $\int_{b-c}^{b+c}, \int_{-c}^{c}$ and the remainder. Choose $c$ large enough so that $\int_{-c}^{c} k>9 / 10$.

As $b$ tends to $\infty, \int_{b-c}^{b+c}$ tends to $+\infty, \int_{-c}^{c}$ stays bounded and the absolute value of the integral over the rest is $<(3 / 10) \int_{b-c}^{b+c}$.

$$
\begin{aligned}
\int f \sum_{i=1}^{n} u^{(i)} & =\int\left(\sum_{i=1}^{n} T^{i}\left(g * \psi_{I}\right)\right) \cdot k=\int\left[\left(\sum_{i=1}^{n} T^{i} g\right) * \psi_{I}\right] k \\
& =\int\left[\left(\sum_{i=1}^{n} T^{i} g_{1}\right) * \psi_{I}\right]\left(k-{ }_{b} k\right) .
\end{aligned}
$$

Now write

$$
\sum_{i=1}^{n} T^{i} g_{1}=\sum_{i=1}^{n} T^{i}(r-h)=\sum_{i=1}^{n} \dot{r}_{i}+\sum_{i=1}^{n} \sum_{j=1}^{n-i} T^{j} l_{i}-\sum_{i=1}^{n} T^{i} h .
$$

What remains to be shown at this point because of (5), (6) and (7) is that

$$
\left|\int\left[\left(\sum_{i=1}^{n} \sum_{j=1}^{n-1} T^{j} l_{i}-\sum_{i=1}^{n} T^{i} h\right) * \psi_{I}\right]\left[k-{ }_{b} k\right]\right|<1
$$

if we fix $b$ and then take $n$ large enough. 
To see (8) note that $\sum_{i=1}^{n} l_{i}=h$. See (C)(1) of T3.2. Pick a $K$ so that

$$
\int\left|\sum_{i=1}^{K} l_{i}-h\right|<\varepsilon
$$

where $\varepsilon$ is chosen so that if $B$ is the bound for $\sum_{i=1}^{m}\left(T^{i} \delta_{x}\right)\left(\psi_{I+b}-\psi_{I}\right)$ given by T3.2 ( $B$ depends on $b$ ) then $\varepsilon B<\frac{1}{2}$. Now rewrite the left side of (8).

$$
\begin{aligned}
\int\left[\left(\sum_{i=1}^{K} \sum_{j=1}^{n-i} T^{j} l_{i}\right.\right. & \left.\left.-\sum_{i=1}^{K} \sum_{j=1}^{n} T^{j} l_{i}\right) * k\right]\left(\psi_{I}-\psi_{I+b}\right) \\
& +\int\left[\left(\sum_{i=K+1}^{n} \sum_{j=1}^{n-i} T^{j} l_{i}-\sum_{i=K+1}^{\infty} \sum_{j=1}^{n} T^{j} l_{i}\right) * k\right]\left(\psi_{I}-\psi_{I+b}\right) .
\end{aligned}
$$

The first term of (9) tends to 0 as $n \rightarrow \infty$ because for each $l_{i}, \int\left[\left(T^{n} l_{i}\right) * \psi_{I}\right] \cdot k$ tends to 0 .

$K$ was chosen so that $\int\left|\sum_{i=K+1}^{\infty} l_{i} * k\right|<\varepsilon$ and because of the way we choose $\varepsilon$ and because of T0.3 the second term of (9) is less than $\frac{1}{2}$.

\section{Section 5}

THEOREM 1. Let $r>0$ be a bounded Borel measurable function with bounded support. Let

$$
l_{n}=C_{n}-\sum_{i=0}^{n} u^{(i)}
$$

$\left(C_{n}=\int_{0}^{1} \sum_{i=0}^{n} u^{(i)}, u^{(0)}=\delta_{0}, u^{(1)}=u, u^{(2)}=u * u\right.$ etc. $)$

Let $l=\lim _{n \rightarrow \infty} l_{n}\left(l_{n}^{\prime \prime} \rightarrow l^{\prime \prime}\right.$ by T3.4; the singular parts obviously converge). Then (a) there is a constant $K$ such that $l * r+K \geqq 0$ everywhere and (b) $u *(l * r)=l * r+r$.

Proof. It will be convenient to assume $\int r=1$. To see (a) note that

$$
\int_{x}^{x+1} \sum_{i=1}^{n} u^{(i)} \leqq \int_{0}^{1} \sum_{i=1}^{n} u^{(i)} * v
$$

where $v$ is the distribution of the first hit in $(x, x+1)$, translated by $-x$.

By T3.2 and T0.3

$$
\int_{0}^{1} \sum_{i=1}^{\infty} u^{(i)}-\int_{0}^{1} \sum_{i=1}^{\infty} u^{i} * v \leqq B
$$

(1) and (2) give (a) for $r=\psi_{(0,1)}$. By T3.2

$$
\left|\left(\sum_{i=1}^{n} u^{(i)}\right) *\left(r-\psi_{(0,1)}\right)\right| \leqq B
$$

everywhere, giving (a). Actually we have proved a little more, namely

$$
l_{n} * r+K>0 \text { everywhere for all } n .
$$


From (3) and the fact that $\lim _{n \rightarrow \infty} u^{(n)} * r=0$ everywhere we get that

$$
u *(l * r) \leqq(l * r)+r .
$$

So far everything has been straightforward. We will now use the same idea as in Spitzer [6], namely: we will show

$$
(l * r)+r-u *(l * r) \text { is a constant a.e. (call it } C) .
$$

$C$ must be 0 since

$$
u^{(n)}(l * r)=l * r+\sum_{i=0}^{n} u^{(i)} r-n C \geqq 0
$$

and $u^{(i)}, r \rightarrow 0$. To show (5) we will proceed as in T3.2. Define $h$ to be $r$ translated by $x$. Define $r_{i}$ and $l_{i}$ as we did there. Then

$$
\sum_{i=1}^{n} u^{(n)} * r=\sum_{i=1}^{n} r_{i}+\sum_{i=1}^{n} \sum_{j=1}^{n-i} u^{(j)} * l_{i}
$$

and

$$
\sum_{i=1}^{\infty} l_{i}=h
$$

by $(C)(1)$ in the proof of $\mathrm{T} 3.2$

$$
\lim _{n \rightarrow \infty}\left(\sum_{i=1}^{n} \sum_{j=1}^{n-i} u^{(j)} * l_{i}-\sum_{j=1}^{n} u^{(j)} * h\right)=C
$$

where $C$ is a constant.

We will devote the next few equations to proving (8).

$$
\lim _{n \rightarrow \infty}\left(C_{n}-\sum_{j=1}^{n} u^{(j)} * h\right)
$$

exists everywhere and the convergence is uniform on finite intervals, by T3.4.

$$
\lim _{n \rightarrow \infty}\left(C_{n}-\sum_{i=1}^{n} \sum_{j=1}^{n-i} u^{(j)} * l_{i}\right)
$$

exists everywhere and the convergence is in $L_{1}$ on finite intervals. This follows from (6) and (9) with $h$ replaced by $r$.

(11) Let $g_{n}$ stand for the expression in (8). Then

$$
\int\left(\lim _{n \rightarrow \infty} g_{n}\right) \cdot f=0
$$

for any bounded function $f$ with bounded support and $\int f=0((11)$ implies (8)). To get (11): note that

$$
\int\left(\lim _{n \rightarrow \infty} g_{n}\right) f=\lim _{n \rightarrow \infty} \int g_{n} \cdot f
$$


because of (9) and (10). Let $g_{n}=c+d$ where

By T3.2

$$
\begin{aligned}
& c=\sum_{i=1}^{K} \sum_{j=1}^{n-i} u^{(j)} * l_{i}-\sum_{i=1}^{K} \sum_{j=1}^{n} u^{(j)} * l_{i} \\
& d=\sum_{i=K+1}^{n} \sum_{j=1}^{n-i} u^{(j)} * l_{i}-\sum_{i=K+1}^{n} \sum_{j=1}^{n} u^{(j)} * l_{i} .
\end{aligned}
$$

$$
\left|\int \sum_{i=1}^{\infty} u^{(i)} * \delta_{x} \cdot f\right|<B \text { for all } x \text { and } n \text {. }
$$

We can therefore, given $\varepsilon$ pick $K$ so that

$$
\int \sum_{i=K+1}^{\infty} l_{i}<\frac{\varepsilon}{B}
$$

and therefore $\left|\int d \cdot f\right|<\varepsilon$. With $K$ fixed we get that for all $n$ large enough $\left|\int c \cdot f\right|<\varepsilon$. This gives (11) and therefore (8).

$$
l * r=\lim _{n \rightarrow \infty}\left(l_{n} * r\right)
$$

and the convergence is uniform on finite intervals. This follows from T3.4.

$$
l * r=\lim _{n \rightarrow \infty}\left(\sum_{i=1}^{n} r_{i}+\sum_{i=1}^{n} \sum_{j=1}^{n-i} u^{(j)} * l_{i}-C_{n}\right) .
$$

This comes from (12) and (6).

$$
l * r=\sum_{i=1}^{\infty} r_{i}+l * h-C .
$$

This comes from (13), (8) and (12) with $r$ replaced by $h$.

$$
\begin{aligned}
u *(l * r)-(l * r)-r\left(u * \sum_{i=1}^{\infty} r_{i}-\sum_{i=1}^{\infty} r_{i}-r\right) & +u *(l * h)-(l * h) \\
& =u *(l * h)-(l * h)-h .
\end{aligned}
$$

The last equality comes from (C)(3) in the proof of T3.2. (15) implies (5) since $h$ is $r$ translated by an arbitrary $x$.

THEOREM 2. Let $u$ be a probability measure with an absolutely continuous component. Assume the walk is recurrent. If $T f-f=T g-g=\phi$ a.e. $(f(x) \geqq 0, \infty>B>$ $\phi(x) \geqq 0$ and the support of $\phi$ is in a finite interval), then $f-g=a x+b$ a.e. If $\int x^{2} u=\infty$, then $f-g=b$ ( $a$ and $b$ are constants).

Proof. We will fix an interval $K$ and divide it into disjoint intervals $J_{i}$ of equal length. We will then show that the two sequences $\int_{J_{i}} f$ and $\int_{J_{i}} g$ are the same up to a linear sequence (and if $\int x^{2} u=\infty$, up to a constant). Since $K$ and $J_{i}$ are arbitrary, this will be enough. (We assume that the $J_{i}$ are indexed in their natural order.) 
(1) $f$ is integrable on any finite interval. This is so because $u^{(2)}$ is bounded away from 0 on some interval.

(2) Define $f_{n}(x)=\inf (f(x), n)$ and define $l_{n}(x)$ so that $T f_{n}=f_{n}+\phi-l_{n}$.

(3) $l_{n} \geqq 0\left(T f_{n}<f_{n}+\phi\right.$ where $f_{n}<n$ and $\left.T f_{n} \leqq n\right)$.

(4) $T^{j} f_{n}=f_{n}+\sum_{i=1}^{j} T^{i} \phi-\sum_{i=1}^{j} T^{i} l_{n}$.

(5) $\int l_{n} \leqq \int \phi$.

If (5) were not true, write $l_{n}=l_{1}+l_{2}$ where $l_{1}$ is bounded with bounded support and $\int l_{1}=\int \phi$, then $\sum_{i=1}^{j} T^{i}\left(\phi-l_{1}\right)$ converges and $\sum_{i=1}^{j} T^{i} l_{2}$ diverges but this contradicts the fact that $\sum_{i=1}^{j} T^{i}\left(\phi-l_{n}\right)<n$ for all $j$ (by (4)).

(6) $T^{i} f_{n}$ tends (as $j \rightarrow \infty, n$ fixed) in $L_{1}$ on any finite interval to a constant. (6) requires a little proof.

$$
\left(T^{i} f_{n}\right)(x)=\int f_{n} \cdot \tilde{T}^{i} \delta_{x}
$$

and

$$
\left(T^{i} f_{n}\right)(x)-\left(T^{i} f_{n}\right)(y)=\int f_{n} \cdot \tilde{T}^{i}\left(\delta_{x}-\delta_{y}\right)
$$

By T0.7 $\lim _{i \rightarrow \infty} \int\left|\tilde{T}^{i}\left(\delta_{x}-\delta_{y}\right)\right|=0$ and since $f_{n}$ is bounded we get (6).

(7) For a fixed interval $I$

$$
\lim _{n \rightarrow \infty} \int_{I} l_{n}=0
$$

(This is so because $l_{n}$ tends to 0 pointwise and $l_{n}<f+\phi$.)

For large $I$ about 0 write $l_{n}$ as $l_{n}=\left[l_{n}\right]_{1}+\left[l_{n}\right]_{2}+\left[l_{n}\right]_{3}$ where $\left[l_{n}\right]_{1}$ is the part of $l_{n}$ in $I,\left[l_{n}\right]_{2}$ the part to the left of $I$. Write $\phi$ as $a \phi+b \phi$ where $a$ and $b$ are positive constants and $\int a \phi=\int\left[l_{n}\right]_{1}$.

(9) By choosing $n$ large enough we could force

$$
\left|\int \sum_{k=1}^{N} T^{k}\left(a \phi-\left[l_{n}\right]_{1}\right)\left(\psi_{J_{i}}-\psi_{J_{i}}\right)\right|<\varepsilon
$$

for all $J_{i}, J_{j}$ and $N$ by (7) and T3.2 ( $I$ is fixed).

(10) If $I$ is large enough $\int_{J_{t}} \sum_{k=1}^{j} T^{k}\left[l_{n}\right]_{2}$ tends (as $j \rightarrow \infty, n$ fixed) to a sequence $a$ (indexed by $i$ ) that is almost linear by T3.1, and T3.2 because

$$
\int \sum_{k=1}^{j} \tilde{T}^{k}\left(\psi_{j_{l}}-\psi_{J_{l-1}}\right)\left[l_{n}\right]_{2}-\int \sum_{k=1}^{j} \tilde{T}^{k}\left(\psi_{J_{i}}-\psi_{J_{i-1}}\right)\left[l_{n}\right]_{2}
$$

tends (as $j \rightarrow \infty, n$ fixed) to $\varepsilon$ where $\varepsilon$ can be made as small as we want by making $I$ large enough. (We assume that the $J_{i}$ are indexed in their natural order.)

$$
\int_{J_{i}} \sum_{k=1}^{j} T^{k} \phi-\int \sum_{J_{0}}^{j} T_{k=1}^{k} \phi
$$

tends to a limit for each $i$ as $j \rightarrow \infty$. 
(12) We can now see that the sequence $\int_{J_{l}} f_{n}$ is determined (up to a linear function, i.e. an arithmetic progression) by $\phi$. Choose $I$ to satisfy (10), then $n$ to satisfy (9). We now use (6), (9), (10), and (11) to evaluate the different terms in (4).

(13) If $\int x^{2} u=\infty$ then the sequences obtained in (10) are almost constant by T3.3.

\section{BIBLIOGRAPHY}

1. K. L. Chung and W. H. J. Fuchs, On the distribution of values of sums of random variables, Mem. Amer. Math. Soc. No. 6 (1951).

2. William Feller, An introduction to probability theory and its applications, Vol. 2, Wiley, New York, 1966.

3. Carl Herz, Les théorèmes de renouvellement, Ann. Inst. Fourier (Grenoble) 15 (1965), 169188.

4. Harry Kesten and Frank Spitzer, Random walk on countably infinite Abelian groups, Acta. Math. 114 (1965), 237-265.

5. Sidney Port and Charles Stone, Hitting times and hitting places for non-lattice recurrent random walks, (to appear).

6. Frank Spitzer, Principles of random walk, Van Nostrand, Princeton, N. J., 1964.

STANFORd UNIVERSITY, Stanford, California 